\title{
Holographic renormalization of 3D minimal massive gravity
}

Mohsen Alishahiha, ${ }^{a}$ Mohammad M. Qaemmaqami, ${ }^{b}$ Ali Naseh ${ }^{b}$ and Ahmad Shirzad ${ }^{c, b}$

${ }^{a}$ School of Physics, Institute for Research in Fundamental Sciences (IPM), P.O. Box 19395-5531, Tehran, Iran

${ }^{b}$ School of Particles and Accelerators, Institute for Research in Fundamental Sciences (IPM), P.O. Box 19395-5531, Tehran, Iran

${ }^{c}$ Department of Physics, Isfahan University of Technology, P.O.Box 84156-83111, Isfahan, IRAN

E-mail: alishah@ipm.ir, m.qaemmaqami@ipm.ir, naseh@ipm.ir, shirzad@ipm.ir

ABSTRACT: We study holographic renormalization of 3D minimal massive gravity using the Chern-Simons-like formulation of the model. We explicitly present Gibbons- Hawking term as well as all counterterms needed to make the action finite in terms of dreibein and spin-connection. This can be used to find correlation functions of stress tensor of holographic dual field theory.

KEYwords: Gauge-gravity correspondence, AdS-CFT Correspondence, Classical Theories of Gravity

ARXiv EPrint: 1511.06194 


\section{Contents}

1 Introduction 1

2 Asymptotic analysis 3

$\begin{array}{llr}3 & \text { Variational principle } & 8\end{array}$

4 MMG on-shell action $\quad 10$

5 Correlation functions 13

6 Conclusions 16

$\begin{array}{ll}\text { A Explicit form of the action } & 17\end{array}$

$\begin{array}{ll}\text { B Divergent terms of on-shell action } & 18\end{array}$

C Coefficients in counterterm action $\quad 20$

$\begin{array}{lll}\text { C.1 } & \sigma=-1 & 20\end{array}$

C.2 $\sigma=1 \quad 21$

D Local finite terms in on-shell action $\quad 21$

D.1 $\sigma=-1 \quad 21$

D.2 $\sigma=1 \quad 21$

E Local terms in one-point functions $\quad 22$

E.1 $\sigma=-1 \quad 22$

E.2 $\sigma=1 \quad 22$

\section{Introduction}

Topologically massive gravity (TMG) is a three dimensional gravity whose action consists of Einstein gravity with a cosmological constant plus the gravitational Chern-Simon term [1, 2]. The model has two free parameters (cosmological constant and the coefficient of ChernSimon term) and for generic values of the parameters the corresponding equations of motion admit several solutions including AdS, BTZ and warped AdS black holes solutions [3-6]. It is, however, known that TMG suffers from the fact that the energy of graviton and the mass of BTZ black holes cannot be positive at the same time (see e.g. [7]).

In order to circumvent the above problem, the authors of [8], proposed a new model, named Minimal Massive Gravity (MMG), which is a ghost free and non-tachyonic three dimensional theory. Indeed the proposed model which is a natural extension of TMG has one more tunable parameter, though the same as TMG, it has a single massive mode above a flat space or an AdS vacuum. 
A peculiar feature of MMG is that it does not have an action in the metric formulation, though it is possible to wire an action in terms of dreibein. More precisely the Lagrangian 3 -form of MMG is [8]

$$
\begin{aligned}
L(e, \omega, h)= & -\sigma e \cdot R(\omega)+\frac{\Lambda_{0}}{6} e \cdot e \times e+h \cdot T(\omega)+\frac{1}{2 \mu}\left(\omega \cdot d \omega+\frac{1}{3} \omega \cdot \omega \times \omega\right) \\
& +\frac{\alpha}{2} e \cdot h \times h
\end{aligned}
$$

where $\sigma$ is a sign, $\Lambda_{0}$ is a cosmological constant, $e$ and $\omega$ are dreibein and dualised spinconnection, respectively. $\mu$ is a mass parameter of the model. Moreover in terms of these variables the Lorentz covariant torsion and curvature 2-forms are given by

$$
T(\omega)=d e+\omega \times e, \quad R(\omega)=d \omega+\frac{1}{2} \omega \times \omega .
$$

Here $h$ is an extra field that for $\alpha=0$ case it may be thought of as a Lagrange multiplier to impose the zero torsion constraint. Indeed in this case the model reduces to the TMG model.

To explore different properties of the model, it is useful to obtain equations of motion derived from the action (1.1). It is, however, important to note that in order to find the equations of motion one should make sure that the boundary terms appearing due to the variation of the action could consistently be removed from the variation. This may be done by imposing proper boundary conditions or/and adding certain boundary terms. It is indeed the aim of this paper to explore this point for MMG model using the holographic renormalization method [9].

We note, however, that since the model does not have an action in the metric formulation one needs to study holographic renormalization of the MMG model in terms of its Chern-Simons like formulation. Holographic renormalization for 3D Einstein gravity and 3D topologically massive gravity in dreibein formalism have been studied in $[10,11]$ and $[12,13]$, respectively. ${ }^{1}$

Different aspects of MMG model have been studied in e.g. [16-26]. In particular it was shown that the model has a critical point at which it exhibits logarithmic solutions. The corresponding solution may provide gravitational descriptions for dual logarithmic conformal field theories. Using the procedure developed [27] two point functions of stress energy and its logarithmic partner have been obtained in [19] where the new anomaly has also been read from the expressions of the corresponding two point functions. Here we will re-derive these results rather rigorously using the holographic renormalization method. To do so, we will have to carefully study variational principle of the model and moreover to find any possible counterterms necessitate to make the model finite.

The paper is organized as follows. In the next section we will consider asymptotic analysis of the equations of motion of MMG model in the first order formalism. This study can be used to examine the validity of the variational principle of the model which is considered in section three. In section four we will study the on-shell action of the

\footnotetext{
${ }^{1}$ See $[14,15]$ for holographic renormalization of other 3D gravities.
} 
model where we will obtain the necessary counterterms to make the action finite. Using the resultant finite on-shell action at the critical point we will obtain two point functions of holographic stress tensor and its logarithmic partner in section five. This result shows that the MMG model at critical point could also provide a holographic description for a logarithmic CFT which is non-unitary. The last section is devoted to conclusions.

\section{Asymptotic analysis}

In this section we would like to further study asymptotic behavior of the linearized equations of motion of MMG model. The linearized equations of motion of the MMG model above an AdS vacuum in the first order formalism have been considered in [8]. In what follows we will first review the the relevant part of the paper [8] and then we will solve the corresponding equations asymptotically in the Fefferman-Graham gauge.

To proceed we note that although for generic $\alpha \neq 0$ the torsion $T(\omega)$ is non-zero, one may define a new torsion free spin connection $\Omega=\omega+\alpha$ by which the Lagrangian 3 -form (1.1) reads [8]

$$
\begin{aligned}
L(e, \Omega, h)= & -\sigma e \cdot R(\Omega)+\frac{\Lambda_{0}}{6} e \cdot e \times e+h \cdot T(\Omega)+\frac{1}{2 \mu}\left(\Omega \cdot d \Omega+\frac{1}{3} \Omega \cdot \Omega \times \Omega\right) \\
& -\frac{1}{2 \mu}\left(\alpha \Omega \cdot D h+\alpha h \cdot R(\Omega)-\alpha^{2} h \cdot D h+\frac{\alpha^{3}}{3} h \cdot h \times h\right) \\
& +\sigma \alpha e \cdot D h-\frac{\alpha}{2}(1+\sigma \alpha) e \cdot h \times h .
\end{aligned}
$$

In this notation, assuming to have a well defined variation principle, the corresponding equations of motion are

$$
\begin{aligned}
& E_{e}=-\sigma R(\Omega)+(1+\sigma \alpha) D h+\frac{\Lambda_{0}}{2} e \times e-\frac{\alpha}{2}(1+\sigma \alpha) h \times h=0, \\
& E_{\Omega}=-\sigma T(\Omega)+(1+\sigma \alpha) e \times h+\frac{1}{2 \mu}\left(2 R(\Omega)+\alpha^{2} h \times h-2 \alpha D h\right)=0, \\
& E_{h}=(1+\sigma \alpha) T(\Omega)-\alpha(1+\sigma \alpha) e \times h+\frac{1}{2 \mu}\left(2 \alpha^{2} D h-2 \alpha R(\Omega)-\alpha^{3} h \times h\right)=0 .
\end{aligned}
$$

Here the covariant derivative is defined by $D A=d A+\Omega \times A$. Using the equations of motion for $\Omega$ and $h$ one can see that $\Omega$ is a torsion free spin-connection,

$$
T(\Omega)=0,
$$

by which the other equations of motion may be recast into the following forms

$$
\begin{array}{r}
\sigma R(\Omega)-(1+\sigma \alpha) D h-\frac{\Lambda_{0}}{2} e \times e+\frac{\alpha}{2}(1+\sigma \alpha) h \times h=0, \\
R(\Omega)-\alpha D h+\mu(1+\sigma \alpha) e \times h+\frac{\alpha^{2}}{2} h \times h=0 .
\end{array}
$$

These equations of motion exhibits an AdS vacuum solution with radius $l$ which can be given in terms of the parameters of the action as follows

$$
\frac{\Lambda_{0}}{\mu^{2}}=-\frac{\sigma}{\mu^{2} l^{2}}+\alpha(1+\sigma \alpha) C^{2}, \quad \text { with } C=-\frac{\left(\alpha \Lambda_{0} l^{2}-1\right)}{2 \mu^{2} l^{2}(1+\sigma \alpha)^{2}} .
$$


Note that for this solution one also gets $h=C \mu e$. It is then natural to study small fluctuations above this solution. Denoting the vacuum solution by $\bar{e}, \bar{\Omega}, \bar{h}$, then a general perturbation may be written as

$$
e=\bar{e}+\mathbf{k}, \quad \Omega=\bar{\Omega}+\mathbf{v}, \quad h=C \mu(\bar{e}+\mathbf{k})+\mathbf{p},
$$

where $\mathbf{k}, \mathbf{v}$ and $\mathbf{p}$ are small perturbations of $e, \Omega$ and $h$ respectively. Plugging this anstatz into the equations of motion given by (2.3) and (2.4) and using (2.5) one arrives at

$$
\bar{D} \mathbf{k}+\bar{e} \times \mathbf{v}=0, \quad \bar{D} \mathbf{v}+\bar{e} \times\left[\mu(1+\sigma \alpha)^{2} \mathbf{p}+\frac{1}{l^{2}} \mathbf{k}\right]=0, \quad \bar{D} \mathbf{p}+M \bar{e} \times \mathbf{p}=0,
$$

where $M=\mu(\sigma(1+\sigma \alpha)-\alpha C)$.

Now the aim is to solve these linearized equations. To do so we will proceed as follows. By making use of the first equation in (2.7) one can find $\mathbf{v}$ in terms of $\mathbf{k}$. More precisely one has

$$
\mathbf{v}_{\mu}^{a}=-(\bar{e})^{-1} \epsilon^{\lambda \rho \nu}\left(\bar{e}_{\mu b} \bar{e}_{\lambda}^{a}-\frac{1}{2} \bar{e}_{\mu}^{a} \bar{e}_{\lambda b}\right) \bar{D}_{\rho} \mathbf{k}_{\nu}^{b} .
$$

Note that this expression is exactly the same as that for 3D Einstein gravity [28]. This is due to the fact that the first equation in (2.7) is, indeed, the torsionless condition appearing in both models.

Solving the second equation of (2.7) for $\mathbf{p}$ gives

$$
\mathbf{p}_{\mu}^{a}=-\frac{1}{\mu(1+\sigma \alpha)^{2}}\left((\bar{e})^{-1} \epsilon^{\lambda \rho \nu}\left(\bar{e}_{\mu b} \bar{e}_{\lambda}^{a}-\frac{1}{2} \bar{e}_{\mu}^{a} \bar{e}_{\lambda b}\right) \bar{D}_{\rho} \mathbf{v}_{\nu}^{b}+\frac{1}{l^{2}} \mathbf{k}_{\mu}^{a}\right),
$$

where $\mathbf{v}$ is given by (2.8). Finally utilizing the expressions of $\mathbf{v}$ and $\mathbf{p}$ and from the last equation of (2.7) one arrives at

$$
\begin{array}{r}
\epsilon^{\lambda \mu \nu} \epsilon^{\gamma \rho \sigma} \epsilon^{\eta \alpha \beta}\left(\bar{e}_{\nu b} \bar{e}_{\gamma}^{a}-\frac{1}{2} \bar{e}_{\nu}^{a} \bar{e}_{\gamma b}\right)\left(\bar{e}_{\sigma c} \bar{e}_{\eta}^{b}-\frac{1}{2} \bar{e}_{\sigma}^{b} \bar{e}_{\eta c}\right) \bar{D}_{\mu} \bar{D}_{\rho} \bar{D}_{\alpha} \mathbf{k}_{\beta}^{c} \\
+M \epsilon^{\lambda \mu \nu} \epsilon^{\gamma \rho \sigma} \epsilon^{\eta \alpha \beta} \varepsilon_{b c}^{a} \bar{e}_{\mu}^{b}\left(\bar{e}_{\nu f} \bar{e}_{\gamma}^{c}-\frac{1}{2} \bar{e}_{\nu}^{c} \bar{e}_{\gamma f}\right)\left(\bar{e}_{\sigma d} \bar{e}_{\eta}^{f}-\frac{1}{2} \bar{e}_{\sigma}^{f} \bar{e}_{\eta d}\right) \bar{D}_{\rho} \bar{D}_{\alpha} \mathbf{k}_{\beta}^{d} \\
-\frac{\bar{e}^{2}}{l^{2}} \epsilon^{\lambda \mu \nu} \bar{D}_{\mu} \mathbf{k}_{\nu}^{a}-\frac{\bar{e}^{2}}{l^{2}} M \epsilon^{\lambda \mu \nu} \varepsilon_{b c}^{a} \bar{e}_{\mu}^{b} \mathbf{k}_{\nu}^{c}=0,
\end{array}
$$

that is a third order differential equation for $\mathbf{k}$. This equation should be compared with the third order differential equation obtained in [8] for $\mathbf{k}_{\mu \nu}=\tilde{e}_{\mu}^{a} \mathbf{k}_{\nu a}$ where it was shown that the corresponding equation can be factorized into three first order differential equations. Of course since we are interested in the holographic renormalization of the model in the dreibein formulation in what follows we will directly work with $\mathbf{k}_{\mu}^{a}$ fields. Moreover in the context of holographic renormalization it is more appropriate to work in the FeffermanGraham coordinates.

Actually an asymptotically locally AdS geometry in the Fefferman-Graham (FG) coordinates may be given as follows ${ }^{2}$

$$
d s^{2}=\frac{d r^{2}}{4 r^{2}}+\frac{1}{r} g_{i j}\left(r, x^{\alpha}\right) d x^{i} d x^{j} .
$$

\footnotetext{
${ }^{2}$ For simplicity from now on we set $l=1$.
} 
It can be verified that a dreibein which could reproduce the above metric in the FG gauge has the following structure [12]

$$
e_{r}^{a}=\left(\frac{1}{2 r}, 0,0\right), \quad e_{i}^{a}=\left(0, e_{i}^{2}, e_{i}^{3}\right)
$$

Here we are using a notation in which $x^{\mu}=\left(r, x^{i}\right)$ and $x^{i} \equiv(u, v)$. For the vacuum $A d S_{3}$ solution with $\bar{g}_{u u}=\bar{g}_{v v}=0, \bar{g}_{u v}=1 / 2$, one has

$$
\bar{e}_{\mu}^{a}=\left(\begin{array}{ccc}
\frac{1}{2 r} & 0 & 0 \\
0 & 0 & \frac{1}{2 r} \\
0 & 1 & 0
\end{array}\right) .
$$

On the other hand for perturbations in spatial directions, $g_{i j}=\eta_{i j}+h_{i j}$, the corresponding perturbations of dreibein respecting the FG gauge are given by

$$
\mathbf{k}_{r}^{a}=(0,0,0), \quad \mathbf{k}_{i}^{a}=\left(0, \mathbf{k}_{i}^{2}, \mathbf{k}_{i}^{3}\right) .
$$

Having expressed the FG gauge in the dreibein formalism it is important to make sure that it has the right number of independent components. Naively from the above expression it seems that $\mathbf{k}_{i}^{a}$ has four independent components. We note, however, that from the integrability conditions of the equations (2.7) as well as the explicit form of the equation (2.10) one can show that both $\mathbf{p}_{\mu \nu}=\bar{e}_{\mu}^{a} \mathbf{p}_{\nu a}$ and $\mathbf{k}_{\mu \nu}=\bar{e}_{\mu}^{a} \mathbf{k}_{\nu a}$ are symmetric tensors indicating that the actual number of independent components are three.

More precisely from the linearized equations of motion (2.7) and using the symmetries of the background $\mathrm{AdS}_{3}$ geometry and integrability conditions one gets

$$
\bar{e}^{a} \bar{e} \cdot \mathbf{p}=0
$$

showing that $\mathbf{p}_{\mu \nu}$ generally is a symmetric tensor.

On the other hand taking into account that the equation (2.10) may be thought of as a vanishing tensor with indices $\mathbf{E}^{\lambda a}$, one may find a new tensor by acting $\bar{e}_{a}^{\eta}$ on it, i.e. $\mathbf{E}^{\eta \lambda}=\bar{e}_{a}^{\eta} \mathbf{E}^{\lambda a}$. It is then easy to see that the equations of motion in the metric formulation is actually given by $\frac{1}{2}\left(\mathbf{E}^{\eta \lambda}+\mathbf{E}^{\lambda \eta}\right)$, on which only the symmetric combination of $\frac{1}{2}\left(\bar{e}_{\mu}^{a} \mathbf{k}_{\nu a}+\bar{e}_{\nu}^{a} \mathbf{k}_{\mu a}\right)$ appears in the equation. Therefore we could conclude that only symmetric part of $\mathbf{k}_{\mu \nu}$ appears as a dynamical variable which in turn indicates that the actual number of independent components of $\mathbf{k}_{i}^{a}$ is three. ${ }^{3}$ These three independent components may be chosen as $\mathbf{k}_{u}^{2}, \mathbf{k}_{v}^{2}, \mathbf{k}_{v}^{3}$. In this notation one has $\mathbf{k}_{u}^{3}=\frac{1}{2 r} \mathbf{k}_{v}^{2}$.

Using this gauge and contracting the equation (2.10) by $\bar{e}_{\lambda a}$ one finds

$$
-\frac{M}{\mu(1+\sigma \alpha)^{2} r}\left(\frac{1}{2} \partial_{v}^{2} \mathbf{k}_{u}^{2}-\partial_{u} \partial_{v} \mathbf{k}_{v}^{2}+\mathbf{k}_{v}^{2 \prime}+r \partial_{u}^{2} \mathbf{k}_{v}^{3}-2 r \mathbf{k}_{v}^{2 \prime \prime}\right)=0,
$$

where by "prime" we denote a derivative with respect to radial coordinate $r$. In fact writing down different components of (2.10) in terms of $\mathbf{k}_{i}^{a}$ gives the following equations up to an

\footnotetext{
${ }^{3}$ We would like to thank Wout Merbis for a discussion on this point.
} 
overall factor of $\frac{1}{\mu(1+\sigma \alpha)^{2}}$,

$$
\begin{aligned}
-\frac{1}{2} \partial_{v}^{2} \mathbf{k}_{u}^{2 \prime}+\partial_{u}^{2} \mathbf{k}_{v}^{3}+M \mathbf{k}_{v}^{2 \prime \prime}+r \partial_{u}^{2} \mathbf{k}_{v}^{3 \prime}=0, \\
2 r \partial_{v} \mathbf{k}_{u}^{2 \prime \prime}-\partial_{u} \mathbf{k}_{v}^{2 \prime}+2 r \partial_{u} \mathbf{k}_{v}^{2 \prime \prime}+\partial_{v} \mathbf{k}_{u}^{2 \prime}-M \partial_{v} \mathbf{k}_{u}^{2 \prime}+M \partial_{u} \mathbf{k}_{v}^{2 \prime}=0 \\
-4 r^{2} \partial_{u} \mathbf{k}_{v}^{3 \prime \prime}+\partial_{v} \mathbf{k}_{v}^{2 \prime}-2 r \partial_{v} \mathbf{k}_{v}^{2 \prime \prime}-2 M r \partial_{u} \mathbf{k}_{v}^{3 \prime}-10 r \partial_{u} \mathbf{k}_{v}^{3 \prime}+M \partial_{v} \mathbf{k}_{v}^{2 \prime}-2(M+1) \partial_{u} \mathbf{k}_{v}^{3}=0 \\
2 r^{2} \partial_{u}^{2} \mathbf{k}_{v}^{3 \prime}-r \partial_{v}^{2} \mathbf{k}_{u}^{2 \prime}+2 r \partial_{u}^{2} \mathbf{k}_{v}^{3}+2 M r \mathbf{k}_{v}^{2 \prime \prime}=0, \\
-4 r^{2} \mathbf{k}_{v}^{3 \prime \prime \prime}+\partial_{v}^{2} \mathbf{k}_{v}^{2 \prime}-2 r \partial_{u} \partial_{v} \mathbf{k}_{v}^{3 \prime}-(2 M+18) r \mathbf{k}_{v}^{3 \prime \prime}-2 \partial_{u} \partial_{v} \mathbf{k}_{v}^{3}-(4 M+12) \mathbf{k}_{v}^{3 \prime}=0, \\
-2 r \mathbf{k}_{u}^{2 \prime \prime \prime}-\partial_{u} \partial_{v} \mathbf{k}_{u}^{2 \prime}+\partial_{u}^{2} \mathbf{k}_{v}^{2 \prime}+(M-3) \mathbf{k}_{u}^{2 \prime \prime}=0 .
\end{aligned}
$$

So far we have presented the full linearized gauged fixed equations of motion of small fluctuations above an AdS vacuum of the MMG model. In what follows we shall study their asymptotic solutions which can be used to determine divergent terms appearing in the on-shell action. This analysis, in turns, may be used to fix the boundary terms needed to have a well-imposed variational principle.

To proceed we solve the equations (2.16) and (2.17) order by order in $r$ near the boundary at $r=0$. Motivated by the pp-wave solution of the model [19], the most general near boundary expansions compatible with the linearized equations of motion can be considered as follows ${ }^{4}$

$$
\begin{aligned}
& \mathbf{k}_{u}^{2}=\chi_{u}^{(0)} \log (r)+\varphi_{u}^{(0)}+\chi_{u}^{(1)} r \log (r)+\varphi_{u}^{(1)} r+\ldots, \\
& \mathbf{k}_{v}^{3}=\frac{1}{2 r}\left(\chi_{v}^{(0)} \log (r)+\varphi_{v}^{(0)}+\chi_{v}^{(1)} r \log (r)+\varphi_{v}^{(1)} r+\ldots\right) \\
& \mathbf{k}_{v}^{2}=\tilde{\chi}_{v}^{(0)} \log (r)+\tilde{\varphi}_{v}^{(0)}+\tilde{\chi}_{v}^{(1)} r \log (r)+\tilde{\varphi}_{v}^{(1)} r+\ldots
\end{aligned}
$$

Plugging these expressions into the equations (2.16) and (2.17) and assuming $(1+\sigma \alpha) \neq 0$ one may solve the equations as follows. Indeed from the equation $(2.16)$ at $\log (r) / r$ order one gets

$$
-\frac{M}{2 \mu}\left(2 \tilde{\chi}_{v}^{(1)}+\partial_{v}^{2} \chi_{u}^{(0)}+\partial_{u}^{2} \chi_{v}^{(0)}-2 \partial_{u} \partial_{v} \tilde{\chi}_{v}^{(0)}\right)=0
$$

while at $1 / r^{2}$ order from (2.16) and the fifth equation in (2.17) one finds respectively

$$
-\frac{3 M}{\mu} \tilde{\chi}_{v}^{(0)}=0, \quad \frac{(M-1)}{\mu} \chi_{v}^{(0)}=0
$$

On the other hand at $\log (r)$ order from the second and third equations of (2.17) one has

$$
\frac{2(M-1)}{\mu}\left(\partial_{v} \chi_{u}^{(1)}-\partial_{u} \tilde{\chi}_{v}^{(1)}\right)=0, \quad \frac{(M+1)}{\mu}\left(\partial_{u} \chi_{v}^{(1)}-\partial_{v} \tilde{\chi}_{v}^{(1)}\right)=0 .
$$

\footnotetext{
${ }^{4}$ Indeed these expansions are analogues to that considered in [29] using metric formulation of TMG.
} 
Moreover from the equation (2.16) and the first, second, third, forth ,fifth and the last equations of (2.17) at order $1 / r$, one finds respectively

$$
\begin{aligned}
& \frac{M}{2 \mu}\left(2 \tilde{\chi}_{v}^{(1)}-2 \tilde{\varphi}_{v}^{(1)}-\partial_{u}^{2} \varphi_{v}^{(0)}-\partial_{v}^{2} \varphi_{u}^{(0)}+2 \partial_{u} \partial_{v} \tilde{\varphi}_{v}^{(0)}\right)=0, \\
& \frac{4 M}{\mu} \tilde{\chi}_{v}^{(0)}=0, \\
& \frac{2}{\mu}\left([M+1] \partial_{v} \chi_{u}^{(0)}-[M-3] \partial_{u} \tilde{\chi}_{v}^{(0)}\right)=0, \\
& \frac{1}{\mu}\left([M-1] \partial_{u} \chi_{v}^{(0)}-[M+3] \partial_{v} \tilde{\chi}_{v}^{(0)}\right)=0,-\frac{2 M}{\mu} \tilde{\chi}_{v}^{(0)} \\
&=0, \\
& \frac{1}{\mu}\left(-[M+1] \chi_{v}^{(1)}+\partial_{v}^{2} \tilde{\chi}_{v}^{(0)}-\partial_{u} \partial_{v} \chi_{v}^{(0)}\right)=0, \\
& \frac{2(M+1)}{\mu} \chi_{u}^{(0)}=0 .
\end{aligned}
$$

Finally from the first, second, third, forth and the last equations of (2.17) at order of one, we find respectively

$$
\begin{aligned}
-\frac{2}{\mu}\left(2 M \tilde{\chi}_{v}^{(1)}+\partial_{u}^{2} \chi_{v}^{(0)}-\partial_{v}^{2} \chi_{u}^{(0)}\right) & =0, \\
\frac{1}{\mu}\left([2 M-6] \partial_{v} \chi_{u}^{(1)}-2[M+1] \partial_{u} \tilde{\chi}_{v}^{(1)}+2(M-1)\left[\partial_{v} \varphi_{u}^{(1)}-\partial_{u} \tilde{\varphi}_{v}^{(1)}\right]\right) & =0, \\
\frac{1}{\mu}\left([1-M] \partial_{v} \tilde{\chi}_{v}^{(1)}+(M+3) \partial_{u} \chi_{v}^{(1)}-(M+1)\left[\partial_{v} \tilde{\varphi}_{v}^{(1)}-\partial_{u} \varphi_{v}^{(1)}\right]\right) & =0, \\
\frac{1}{\mu}\left(2 M \tilde{\chi}_{v}^{(1)}+\partial_{u}^{2} \chi_{v}^{(0)}-\partial_{v}^{2} \chi_{u}^{(0)}\right) & =0, \\
\frac{2}{\mu}\left([1-M] \chi_{u}^{(1)}-\partial_{u}^{2} \tilde{\chi}_{v}^{(0)}+\partial_{u} \partial_{v} \chi_{u}^{(0)}\right) & =0 .
\end{aligned}
$$

Now the aim is to solve these equations to find different components appearing in the asymptotic expansions (2.18). In particular for the cases of $(\alpha=0, \mu \rightarrow \infty)$ and $(\alpha \neq$ $0, \mu \rightarrow \infty)$ where $M \rightarrow \infty$ from equations (2.19)-(2.23) one arrives at

$$
\begin{aligned}
\chi_{u}^{(0)}=\chi_{v}^{(0)}=\tilde{\chi}_{v}^{(0)}=\chi_{u}^{(1)}=\chi_{v}^{(1)}=\tilde{\chi}_{v}^{(1)}=0, & \tilde{\varphi}_{v}^{(1)}=-\frac{1}{8} R\left[\varphi^{(0)}\right], \\
\partial_{v} \tilde{\varphi}_{v}^{(1)}-\partial_{u} \varphi_{v}^{(1)}=0, & \partial_{v} \varphi_{u}^{(1)}-\partial_{u} \tilde{\varphi}_{v}^{(1)}=0,
\end{aligned}
$$

where $R\left[\varphi^{(0)}\right]=4\left(\partial_{u}^{2} \varphi_{v}^{(0)}+\partial_{v}^{2} \varphi_{u}^{(0)}-2 \partial_{u} \partial_{v} \tilde{\varphi}_{v}^{(0)}\right)$. It is worth noting that these results are in agreement with that obtained from metric formulation for 3D Einstein gravity [30]. Note also that since the MMG model without the Chern-Simons term is the same as 3D Einstein gravity, these results show that adding $\alpha$-term would not change the content of the 3D Einstein gravity. 
On the other hand for $\alpha=-\frac{2}{\mu}(1+\mu \sigma)$ where one has $M=-1$, from the above equations, one gets

$$
\begin{aligned}
\chi_{v}^{(0)} & =\tilde{\chi}_{v}^{(0)}=0, & \chi_{u}^{(1)} & =-\frac{1}{2} \partial_{u} \partial_{v} \chi_{u} \\
\tilde{\chi}_{v}^{(1)} & =-\frac{1}{8} R\left[\chi^{(0)}\right], & \partial_{u} \chi_{v}^{(1)} & =-\partial_{v} \tilde{\chi}_{v}^{(1)}, \\
\tilde{\varphi}_{v}^{(1)} & =\tilde{\chi}_{v}^{(1)}-\frac{1}{8} R\left[\varphi^{(0)}\right], & \partial_{v} \chi_{u}^{(1)}+\left(\partial_{v} \varphi_{u}^{(1)}-\partial_{u} \tilde{\varphi}_{v}^{(1)}\right) & =0 .
\end{aligned}
$$

Here we have used the identity $R\left[\chi^{(0)}\right]=4 \partial_{v}^{2} \chi_{u}^{(0)}$.

\section{Variational principle}

In the previous section we studied asymptotic solutions of the linearized equations of motion of the MMG model. Of course the results rely on the fact that the model admits a wellimposed variational principle. This procedure requires proper boundary terms to make sure that all boundary terms can be consistently removed. In this section we would like to reexamine the variation of the action leading to the corresponding equations of motion.

To proceed let us consider the action of the MMG model (2.1) whose variation with respect to the fields $e, \Omega$ and $h$ are given by

$$
\begin{aligned}
& \delta_{e} L(e, \Omega, h)=E_{e} \cdot \delta e-D(\Omega)(h \cdot \delta e) \\
& \delta_{\Omega} L(e, \Omega, h)=E_{\Omega} \cdot \delta \Omega+D(\Omega)\left(\sigma e \cdot \delta \Omega-\frac{1}{2 \mu}(\Omega \cdot \delta \Omega-\alpha h \cdot \delta \Omega)\right), \\
& \delta_{h} L(e, \Omega, h)=E_{h} \cdot \delta h+D(\Omega)\left(-\sigma \alpha e \cdot \delta h-\frac{1}{2 \mu}\left(\alpha^{2} h \cdot \delta h-\alpha \Omega \cdot \delta h\right)\right) .
\end{aligned}
$$

1 Using the Stokes' theorem ${ }^{5}$ the corresponding boundary terms appearing in the above variation may be recast to the following form

$$
\begin{aligned}
\left.\delta S\right|_{\text {boundary }}=\int_{\partial \mathcal{M}} d^{2} x \epsilon^{i j}( & {\left[-C \mu \alpha \sigma e+\frac{C \alpha}{2} \Omega-\left(1+\frac{C \alpha^{2}}{2}\right) h\right]_{i a} \delta e_{j}^{a} } \\
+ & {\left.\left[\sigma e-\frac{1}{2 \mu} \Omega+\frac{\alpha}{2 \mu} h\right]_{i a} \delta \Omega_{j}^{a}+\left[-\alpha \sigma e+\frac{\alpha}{2 \mu} \Omega-\frac{\alpha^{2}}{2 \mu} h\right]_{i a} \delta \mathbf{p}_{j}^{a}\right), }
\end{aligned}
$$

where we have used $\delta h=C \mu \delta e+\delta \mathbf{p}$ from eq. (2.6).

From the equations (2.8) and (2.9) one observes that the first and second radial derivative of the dreibein appear in the boundary terms. Hence, imposing the Dirichlet boundary condition is not enough to remove all boundary terms. Indeed this is expected simply because even in the Einstein gravity one needs to add a proper Gibbons-Hawking term. In our notation the corresponding Gibbons-Hawking term is

$$
S_{G H}=2 \sigma \mathcal{K}=-\sigma \int_{\partial \mathcal{M}} d^{2} x \epsilon^{i j} \tilde{e}_{i a} \tilde{\Omega}_{j}^{a}
$$

\footnotetext{
${ }^{5}$ The normal vector to the boundary is $n_{\mu}=\left(-\frac{1}{2 r}, 0,0\right)$.
} 
where $\tilde{e}$ and $\tilde{\Omega}$ are boundary dreibein and spin connection respectively. We note, however that, as it is evident from eq.(3.2), the above Gibbons-Hawking term is not sufficient to have a well-defined variational principle and, indeed, more boundary terms are needed. Of course, in general, determining these extra terms is not an easy task. Nonetheless for the model under consideration and for particular values of the parameter, one can fix the boundary terms as follows.

Before proceeding, it is illustrative to take a closer look at the boundary term containing the variation of $\delta \Omega_{j}^{a}$ in (3.2) which includes a variation of radial derivative of dreibein. In the framework of the metric formulation it also contains a variation of radial derivative of boundary metric, $\delta \partial_{r} g_{i j}$. Let us first consider the case of $\alpha=0$, where MMG reduces to TMG. In this case utilizing the metric formulation of TMG it was shown that the contribution of Chern-Simons action (CS) to the coefficient of $\delta \partial_{r} g_{i j}$ vanishes for asymptotically locally AdS space-times (AlAdS) [31]. However, one can see that in the dreibein formulation the contribution of CS action to the coefficient of $\delta \Omega_{j}^{a}$ remains non-zero. This is, indeed, due to the fact that the variation of the CS action in metric and dreibein formalisms are different by certain boundary terms (see eq. (3.23) of ref. [31]). Therefore to have a well-defined variational principle for TMG in dreibein formalism, besides the standard Gibbons-Hawking term (3.3), one needs to add extra boundary terms.

It is worth noting that recently the variational principle of TMG for AlAdS space-times in dreibein formalism is studied in ref. [13]. There it is shown that just half of the standard Gibbons-Hawking term (3.3) should be added to the action in order to have a well-defined variational principle. Actually as it is discussed in refs. [11, 32] there are two possible ways to make the variational principle well defined in dreibein formalism for AlAdS spacetimes. In the first way (the standard case) one imposes the standard Dirichlet boundary condition on the dreibein. In the second way we should impose mixed boundary conditions on both boundary dreibein and its first radial derivative. The study in ref. [13] is based on the second option while in this paper we used the first option for AlAdS space-times. Of course when $\alpha \neq 0$, the coefficients of $\delta \Omega_{j}^{a}$ and $\delta \mathbf{p}_{j}^{a}$ remain non-zero for AlAdS spacetimes. In this case one can remove the $\sigma$-term in coefficient of $\delta \Omega_{j}^{a}$ by adding the standard Gibbons-Hawking term (3.3). Therefore when $\alpha \neq 0$, one needs extra boundary terms to have a well-defined variational principle for AlAdS space-times.

In this paper our main interest is the case $M=-1$, where the model exhibits logarithmic solutions. For this case to determine the solution we will have to know the values of dreibein and its first radial derivative at the boundary. Therefore a well-imposed variational principle occurs by imposing boundary conditions on dreibein and its first radial derivative, simultaneously. Note that this is not the same as that in ref. [13]. This is because in that work the variational principle is studied for AlAdS space-times while the logarithmic solutions are not AlAdS space-times. With this treatment although $\delta \Omega_{j}^{a}$ contains the first radial derivative, the corresponding term does not invalidate the modified variational principle. Nonetheless one still needs to deal with the last term in eq. (3.2) that contains the variation $\delta \mathbf{p}_{i}^{a}$.

Actually we note that different components of $\mathbf{p}_{i}^{a}$ contain the second radial derivative of dreibein and thus have a potential to invalidate even the modified variational principle. 
More precisely one has

$$
\delta \mathbf{p}_{u}^{2}=\frac{4 r^{2}}{\mu(1+\sigma \alpha)^{2}} \delta \mathbf{k}_{u}^{2 \prime \prime}, \quad \delta \mathbf{p}_{v}^{3}=\frac{4 r^{2}}{\mu(1+\sigma \alpha)^{2}} \delta \mathbf{k}_{v}^{3 \prime \prime}+\frac{8 r}{\mu(1+\sigma \alpha)^{2}} \delta \mathbf{k}_{v}^{3 \prime} .
$$

Therefore the corresponding terms which could invalidate the modified variational principle are

$$
\left[-\alpha \sigma e+\frac{\alpha}{2 \mu} \Omega-\frac{\alpha^{2}}{2 \mu} h\right]_{v 2} \delta \mathbf{p}_{u}^{2}-\left[-\alpha \sigma e+\frac{\alpha}{2 \mu} \Omega-\frac{\alpha^{2}}{2 \mu} h\right]_{u 3} \delta \mathbf{p}_{v}^{3} .
$$

By making use of the asymptotic analysis given in the equation (2.25) we get the following asymptotic behavior for $\mathbf{k}$

$$
\begin{aligned}
& \mathbf{k}_{u}^{2}=\chi_{u}^{(0)} \log (r)+\varphi_{u}^{(0)}+\chi_{u}^{(1)} r \log (r)+\varphi_{u}^{(1)} r+\cdots, \\
& \mathbf{k}_{v}^{3}=\frac{1}{2 r}\left(\varphi_{v}^{(0)}+\chi_{v}^{(1)} r \log (r)+\varphi_{v}^{(1)} r+\cdots\right) \\
& \mathbf{k}_{v}^{2}=\tilde{\varphi}_{v}^{(0)}+\tilde{\chi}_{v}^{(1)} r \log (r)+\tilde{\varphi}_{v}^{(1)} r+\cdots
\end{aligned}
$$

Plugging these asymptotic expressions in (3.5) and using eqs. (3.4), (2.8) and (2.9) one arrives at

$$
\mathcal{O}\left(r^{2}\right) \delta \mathbf{k}_{u}^{2 \prime \prime}+\mathcal{O}(r) \delta \mathbf{k}_{v}^{3 \prime \prime}
$$

showing that the boundary terms (3.5) vanish near the boundary and thus would not invalidate the modified variational principle. To summarize, we note that for the MMG model at the critical point one just needs to add the usual Gibbons-Hawking term to have a well-defined modified variational principle. Of course to find a finite on-shell action we will have to add certain counterterms which we will discuss in the following section.

\section{MMG on-shell action}

In this section we would like to compute the on-shell action from which one may evaluate the expectation values of boundary operators using AdS/CFT correspondence. In this context, higher order correlation functions can also be obtained by differentiating the onshell action with respect to the boundary sources.

Using the results of the previous section the total action of MMG is given by

$$
S=S_{M M G}+S_{G H},
$$

which is guaranteed that the model has a well defined variational principle. Nonetheless it is however important to note that even with the Gibbons-Hawking term the on-shell action might diverge and in order to remove the divergent terms one needs to add proper boundary counterterms. This is the aim of this section to determine the divergent terms and then the proper boundary counterterms.

To regularize the on-shell action one can introduces a UV cut-off at $r \geq \epsilon$ and finds the corresponding boundary terms at $r=\epsilon$. Let us expand the regularized on-shell action in power of the fields as follows

$$
\left.S_{\text {on-shell }}\right|_{\text {reg }}=S^{(0)}+S^{(1)}+S^{(2)}+\mathcal{O}\left(\text { fields }^{3}\right),
$$


where by $S^{(0)}, S^{(1)}$ and $S^{(2)}$ are zeroth order (background), linearized and quadratic actions of the perturbations, respectively. It is then straightforward, though tedious, to compute divergent terms of each part. The final results may be summarized as follows

$$
\begin{aligned}
S_{d i v}^{(0)} & =\int d^{2} x \frac{1}{\epsilon} \mathcal{S}_{1 / \epsilon}^{(0)}, \\
S_{d i v}^{(1)} & =\int d^{2} x\left(\log ^{2}(\epsilon) \mathcal{S}_{\log ^{2}(\epsilon)}^{(1)}+\log (\epsilon) \mathcal{S}_{\log (\epsilon)}^{(1)}+\frac{1}{\epsilon} \mathcal{S}_{1 / \epsilon}^{(1)}\right), \\
S_{d i v}^{(2)} & =\int d^{2} x\left(\log ^{3}(\epsilon) \mathcal{S}_{\log ^{3}(\epsilon)}^{(2)}+\log ^{2}(\epsilon) \mathcal{S}_{\log ^{2}(\epsilon)}^{(2)}+\frac{\log (\epsilon)}{\epsilon} \mathcal{S}_{\log (\epsilon) / \epsilon}^{(2)}+\log (\epsilon) \mathcal{S}_{\log (\epsilon)}^{(2)}+\frac{1}{\epsilon} \mathcal{S}_{1 / \epsilon}^{(2)}\right) .
\end{aligned}
$$

The explicit expressions of the coefficients in the above equation are presented in the appendix B. In what follows we will study these divergent terms for the cases $(\alpha=0, \mu \rightarrow$ $\infty),(\alpha \neq 0, \mu \rightarrow \infty)$ and $\left(\alpha=-\frac{2}{\mu}(1+\mu \sigma)\right)$, separately.

Actually for the cases $(\alpha=0, \mu \rightarrow \infty)$ and $(\alpha \neq 0, \mu \rightarrow \infty)$, from the explicit expressions given in the appendix B one gets, ${ }^{6}$

$$
\mathcal{S}_{1 / r}^{(0)}=\sigma, \quad \mathcal{S}_{\log (r)}^{(1)}=-\frac{\sigma}{4} R\left[\varphi^{(0)}\right], \quad \mathcal{S}_{1 / r}^{(1)}=2 \sigma \tilde{\varphi}_{v}^{(0)}, \quad \mathcal{S}_{1 / r}^{(2)}=-\sigma\left(\varphi_{u}^{(0)} \varphi_{v}^{(0)}-\tilde{\varphi}_{v}^{(0) 2}\right)
$$

Hence, the proper boundary counterterms needed to remove these divergent terms are

$$
S_{c t}=2 \sigma \int d^{2} x \tilde{e}+\frac{\sigma}{2} \int d^{2} x R[\tilde{e}] \log (\epsilon)
$$

where $R[\tilde{e}]=-\frac{1}{2} \epsilon^{i j} \partial_{i}\left(\frac{1}{\tilde{e}} \epsilon^{k l} \tilde{e}_{j}^{a} \partial_{k} \tilde{e}_{l a}\right)$ is scalar curvature of 2D boundary geometry. Then the renormalized on shell action becomes

$$
S_{r e n}=\sigma \int d^{2} x\left(\varphi_{u}^{(0)} \varphi_{v}^{(1)}+\varphi_{v}^{(0)} \varphi_{u}^{(1)}+\frac{1}{4} \tilde{\varphi}_{v}^{(0)} R\left[\varphi^{(0)}\right]\right)
$$

in agreement with ref. [30] where the corresponding renormalized on-shell action has been found in the metric formalism. It is worth noting that the form of divergences as well as the renormalized on-shell action for both cases $(\alpha=0, \mu \rightarrow \infty)$ and $(\alpha \neq 0, \mu \rightarrow \infty)$ are the same; indicating that these two models might have the same physical contents.

On the other hand using the expressions presented in the appendix B for $\alpha=-\frac{2}{\mu}(1+$ $\mu \sigma)$ one arrives at $^{7}$

$$
\begin{aligned}
\mathcal{S}_{1 / r}^{(0)} & =\frac{1}{4 \mu}(5 \mu \sigma+1), \\
\mathcal{S}_{\log ^{2}(r)}^{(1)} & =-\frac{3}{32} \frac{\mu \sigma-1}{\mu} R\left[\chi^{(0)}\right], \\
\mathcal{S}_{\log (r)}^{(1)} & =-\frac{3 \sigma \mu^{3}+11 \mu^{2}-12}{16 \mu(2+\mu \sigma)^{2}} R\left[\chi^{(0)}\right]-\frac{3}{16} \frac{\mu \sigma-1}{\mu} R\left[\varphi^{(0)}\right], \\
\mathcal{S}_{1 / r}^{(1)} & =\frac{5 \mu \sigma+1}{2 \mu} \tilde{\varphi}_{v}^{(0)},
\end{aligned}
$$

\footnotetext{
${ }^{6}$ To be precise, for MMG in these two cases we have used eqs. (2.24) instead of (2.25). Actually in these cases the equations in (2.25) reduce to that in (2.24) except for the last equations.

${ }^{7}$ For this case we have $\Lambda_{0}=\frac{1}{2} \mu(\mu \sigma+3)$ and $C=\frac{1}{2}$.
} 


$$
\begin{aligned}
\mathcal{S}_{\log ^{3}(r)}^{(2)}= & \frac{\mu \sigma+1}{12 \mu} \chi_{u}^{(0)} \chi_{v}^{(1)}, \\
\mathcal{S}_{\log (r) / r}^{(2)}= & -\frac{5 \mu \sigma+1}{4 \mu} \chi_{u}^{(0)} \varphi_{v}^{(0)}, \\
\mathcal{S}_{\log ^{2}(r)}^{(2)}= & \frac{\mu \sigma+1}{8 \mu}\left(\varphi_{u}^{(0)} \chi_{v}^{(1)}+\chi_{u}^{(0)} \varphi_{v}^{(1)}-\chi_{u}^{(0)} \partial_{v}^{2} \tilde{\varphi}_{v}^{(0)}+\frac{3}{2} \chi_{u}^{(0)} \partial_{u} \partial_{v} \varphi_{v}^{(0)}\right), \\
\mathcal{S}_{\log (r)}^{(2)}= & \frac{(\mu \sigma+1)}{\mu(2+\mu \sigma)^{2}}\left[\frac{3 \sigma \mu^{3}+25 \mu^{2}+16 \mu \sigma-4}{1+\mu \sigma} \chi_{u}^{(0)} \chi_{v}^{(1)}-2\left(\chi_{u}^{(0)} \varphi_{v}^{(1)}-\varphi_{u}^{(0)} \chi_{v}^{(1)}\right)\right. \\
& +\frac{1}{4}(2+\mu \sigma)^{2}\left(\varphi_{u}^{(0)} \varphi_{v}^{(1)}-3 \varphi_{v}^{(0)} \varphi_{u}^{(1)}-\frac{1}{4} \tilde{\varphi}_{v}^{(0)} R\left[\varphi^{(0)}\right]\right) \\
& \left.-\frac{3}{4}\left(\mu^{2}+4 \mu \sigma-4\right) \chi_{u}^{(0)} \partial_{v}^{2} \tilde{\varphi}_{v}^{(0)}+\frac{1}{2}\left(\mu^{2}+4 \mu \sigma+2\right) \chi_{u}^{(0)} \partial_{u} \partial_{v} \varphi_{v}^{(0)}\right], \\
\mathcal{S}_{1 / r}^{(2)}= & \frac{7 \sigma \mu^{3}+55 \mu^{2}+48 \mu \sigma+4}{4 \mu(2+\mu \sigma)^{2}} \chi_{u}^{(0)} \varphi_{v}^{(0)}-\frac{5 \mu \sigma+1}{4 \mu}\left(\varphi_{u}^{(0)} \varphi_{v}^{(0)}-\tilde{\varphi}_{v}^{(0) 2}\right) .
\end{aligned}
$$

Before proceeding to write proper counterterms to remove the above divergent terms, let us explore certain features of these divergent terms. We note that although for $\sigma=-1, \mu=1$ the equations of motion of critical MMG reduce to that of TMG at the critical point, the above divergent terms which are obtained in the dreibein formalism do not reduce to divergent terms obtained from the metric formalism [29]. This may be understood as follows. Actually the Chern-Simons action in the dreibein formalism differs from ChernSimons action in the metric formalism by certain terms whose variations are boundary terms. Therefore their on-shell values should also be different by these boundary terms (see e.g [31]).

We note also that the above divergent terms contain several terms such as $\chi_{u}^{(0)} \chi_{v}^{(1)}$, whose corresponding counterterms needed to remove them include the dreibein as well as its radial derivative. Of course these types of boundary counteretrms are not allowed for pure 3D Einstein gravity, though they are consistent with MMG at the critical point, as discussed in section 3 .

Finally as it is evident from expressions of (4.7) for a fixed value of $\mu$, the divergent terms change as one changes $\sigma=1$ to $\sigma=-1$. Therefore the corresponding counterterms should be considered separately. Actually it can be seen that in order to remove divergent terms coming from the zeroth and first order of the perturbations one needs to add the following counterterms

$$
S_{c t}^{1}=\int d^{2} x\left(-\frac{5 \sigma \mu+1}{2 \mu} \tilde{e}-\frac{3}{8} \frac{\mu+1}{\mu} R[\tilde{e}] \log (\epsilon)\right),
$$

while the proper counterterms required to remove the divergent terms at second order of perturbations for $\sigma=-1$ are given by

$$
\begin{aligned}
S_{c t}^{2}= & \int d^{2} x\left[a_{1} \mathbf{p} \star \mathbf{k}+a_{2} \mathbf{k} \cdot \mathbf{k}+a_{3} \mathbf{k} \cdot \mathbf{v}+a_{4} \mathbf{v} \cdot \mathbf{v}+a_{5} \mathbf{p} \star \mathbf{v}+a_{6} \mathbf{p} \cdot \mathbf{v}-4 a_{7} \mathbf{k}^{i j} \square \mathbf{k}_{i j}\right. \\
& +\left(b_{1} \mathbf{p} \star \mathbf{k}+b_{2} \mathbf{p} \cdot \mathbf{k}+b_{3} \mathbf{k} \star \mathbf{v}+b_{4} \mathbf{k} \cdot \mathbf{v}+b_{5} \mathbf{p} \star \mathbf{v}+b_{6} \mathbf{p} \cdot \mathbf{v}+b_{7} \mathbf{p} \cdot \mathbf{p}+b_{8} \tilde{e}^{(2)}\right) \log (\epsilon) \\
& \left.+\left(c_{1} \mathbf{p} \star \mathbf{k}+c_{2} \mathbf{p} \cdot \mathbf{k}+c_{3} \mathbf{p} \cdot \mathbf{v}+c_{4} \mathbf{p} \cdot \mathbf{p}+\frac{1}{2} c_{5} \mathcal{K}_{i j} \mathcal{K}^{i j}\right) \log ^{2}(\epsilon)+d_{1} \mathbf{p} \cdot \mathbf{p} \log ^{3}(\epsilon)\right] .
\end{aligned}
$$


However, for $\sigma=1$ they are

$$
\begin{aligned}
S_{c t}^{2}= & \int d^{2} x\left[a_{1} \mathbf{p} \star \mathbf{k}+a_{2} \mathbf{k} \cdot \mathbf{k}+a_{3} \mathbf{p} \cdot \mathbf{k}+a_{4} \mathbf{k} \cdot \mathbf{v}+a_{5} \mathbf{v} \cdot \mathbf{v}+a_{6} \mathbf{p} \star \mathbf{v}-4 a_{7} \mathbf{k}^{i j} \square \mathbf{k}_{i j}\right. \\
& +\left(b_{1} \mathbf{p} \star \mathbf{k}+b_{2} \mathbf{p} \cdot \mathbf{k}+b_{3} \mathbf{k} \star \mathbf{v}+b_{4} \mathbf{k} \cdot \mathbf{v}+b_{5} \mathbf{p} \star \mathbf{v}+b_{6} \mathbf{p} \cdot \mathbf{v}+b_{7} \tilde{e}^{(2)}+\frac{1}{2} b_{8} \mathcal{K}_{i j} \mathcal{K}^{i j}\right) \log (\epsilon) \\
& \left.+\left(c_{1} \mathbf{p} \star \mathbf{k}+c_{2} \mathbf{p} \cdot \mathbf{k}+c_{3} \mathbf{p} \cdot \mathbf{v}+c_{4} \mathbf{p} \cdot \mathbf{p}+\frac{1}{2} c_{5} \mathcal{K}_{i j} \mathcal{K}^{i j}\right) \log ^{2}(\epsilon)+d_{1} \mathbf{p} \cdot \mathbf{p} \log ^{3}(\epsilon)\right] \cdot
\end{aligned}
$$

Here " $\star$ " and "." products are defined by $\mathbf{A} \star \mathbf{B}=\epsilon^{i j} \mathbf{A}_{i}^{a} \mathbf{B}_{j a}, \mathbf{A} \cdot \mathbf{B}=\eta^{i j} \mathbf{A}_{i}^{a} \mathbf{B}_{j a}$, respectively. Moreover $\mathbf{k}_{i j}=\bar{e}_{i}^{a} \mathbf{k}_{j a}, \bar{\square}$ is defined by $2 \mathrm{D}$ flat metric, $\tilde{e}^{(2)}$ is the second order in perturbations of boundary dreibein determinant and $\mathcal{K}_{i j}$ is the first order in perturbations of boundary extrinsic curvature. The explicit values of the coefficients in the above expressions for $\sigma=-1$ and $\sigma=1$ are given in the appendix $\mathrm{C}$.

Adding these counterterms to the on-shell action, all divergent terms given in the equation (4.7) will be removed leading to the following renormalized on-shell action ${ }^{8}$

$$
S_{r e n}=\int d^{2} x\left[\frac{5 \mu+\sigma+8}{2 \mu} \chi_{u}^{(0)} \chi_{v}^{(1)}-\frac{4 \mu}{(\sigma \mu+2)^{2}} \chi_{u}^{(0)} \varphi_{v}^{(1)}-\frac{4 \mu}{(\sigma \mu+2)^{2}} \varphi_{u}^{(0)} \chi_{v}^{(1)}-\frac{4}{\mu} \varphi_{v}^{(0)} \varphi_{u}^{(1)}\right] .
$$

It is worth noting that the above counterterms contain the first and second radial derivatives of the perturbations which in general could destroy the variational principle. We note, however, that following our discussions in the previous section the first radial derivative can be accommodated by the modified boundary condition at the critical point and moreover it can be shown that those terms with the second radial derivative vanish as one approaches the boundary. Therefore the resultant counterterms are consistent with our variational principle.

\section{Correlation functions}

Having found the renormalized on-shell action it is now possible to compute correlation functions of the holographic stress tensor. Following AdS/CFT correspondence the nonnormalizable modes of a bulk field can be identified as a source for a dual operator. Moreover the $n^{\text {th }}$ correlation functions of the dual operator can be evaluated by taking $n^{\text {th }}$ functional derivative of the on-shell action with respect to the source.

In the present case the corresponding sources are $\varphi_{u}^{(0)}, \varphi_{v}^{(0)}, \tilde{\varphi}_{v}^{(0)}$ and $\chi_{u}^{(0)}$ and therefore one point functions of the associated dual operators are given by. ${ }^{9}$

$$
\left\langle T_{i j}\right\rangle=2 \pi\left(\bar{e}_{i a} \frac{\delta S_{r e n}}{\delta e_{a}^{(0) j}}+i \leftrightarrow j\right), \quad\left\langle t_{i j}\right\rangle=-2 \pi\left(\bar{e}_{i a} \frac{\delta S_{r e n}}{\delta \chi_{a}^{(0) j}}+i \leftrightarrow j\right) .
$$

Here we have use the fact that $e_{3}^{(0) v} \equiv 2 \varphi_{u}^{(0)}, e_{2}^{(0) u} \equiv 2 \varphi_{v}^{(0)}, e_{3}^{(0) u} \equiv 2 \tilde{\varphi}_{v}^{(0)}$ and $\chi_{3}^{(0) v} \equiv 2 \chi_{u}^{(0)}$.

\footnotetext{
${ }^{8}$ The renormalized on-shell action also contains local terms whose contributions to the correlation functions are contact terms. Note that these local terms can be changed by adding finite local functions. The explicit forms of these local terms are presented in the appendix D.

${ }^{9}$ The overall factor in action is $1 / 16 \pi G$ and for normalization of one-point and two-point functions we have used the same convention as in ref. [29]. Note also that the sign difference between the definition $\left\langle T_{i j}\right\rangle$ and $\left\langle t_{i j}\right\rangle$ may be understood from the changing the sign of perturbations by raising or lowering the indices.
} 
Using the explicit form of the on-shell action given in the equation (4.11) one can evaluate the one-point functions as follows

$$
\begin{aligned}
\left\langle T_{u u}\right\rangle & =-\frac{1}{2 \mu G} \varphi_{u}^{(1)}+\text { local, } \\
\left\langle T_{v v}\right\rangle & =-\frac{1}{2 G} \frac{\mu}{(\mu \sigma+2)^{2}} \chi_{v}^{(1)}+\text { local, } \\
\left\langle T_{u v}\right\rangle & =\text { local, } \\
\left\langle t_{v v}\right\rangle & =\frac{1}{8 G}\left(-\frac{5 \mu+\sigma+8}{2 \mu} \chi_{v}^{(1)}+\frac{4 \mu}{(\mu \sigma+2)^{2}} \varphi_{v}^{(1)}\right)+\text { local, }
\end{aligned}
$$

where the "local" terms are local functions of sources whose contributions to two-point functions are contact terms. The explicit expressions of these local terms are given in the appendix E.

Using AdS/CFT procedure the higher point correlation functions could also be computed as follows

$$
\begin{aligned}
& \left\langle T_{i j} \cdots\right\rangle=-2 \pi i\left(\bar{e}_{i a} \frac{\delta}{\delta e_{a}^{(0) j}}+i \leftrightarrow j\right)\langle\cdots\rangle, \\
& \left\langle t_{i j} \cdots\right\rangle=2 \pi i\left(\bar{e}_{i a} \frac{\delta}{\delta \chi_{a}^{(0) j}}+i \leftrightarrow j\right)\langle\cdots\rangle .
\end{aligned}
$$

It is important to note that in order to find the higher order correlation functions one should know the explicit dependence of the response functions on the sources. Actually this explicit form cannot be found from the asymptotic analysis of the equations of motion. Indeed in order to find this dependence one needs to solve exactly the linearized equations of motion (2.17) and (2.16) in the bulk. In general it is not an easy task to solve the corresponding linearized equations and it might not even have an explicit analytic solution. Fortunately in the preset case these equations at the critical point $M=-1$ can be solve analytically.

Actually at critical value $M=-1$, the equations (2.16) and (2.17) reduce to

$$
\begin{aligned}
\frac{1}{2} \partial_{v}^{2} \mathbf{k}_{u}^{2}-\partial_{u} \partial_{v} \mathbf{k}_{v}^{2}+\mathbf{k}_{v}^{2 \prime}+r \partial_{u}^{2} \mathbf{k}_{v}^{3}-2 r \mathbf{k}_{v}^{2 \prime \prime}=0 \\
-\frac{1}{2} \partial_{v}^{2} \mathbf{k}_{u}^{2 \prime}+\partial_{u}^{2} \mathbf{k}_{v}^{3}-\mathbf{k}_{v}^{2 \prime \prime}+r \partial_{u}^{2} \mathbf{k}_{v}^{3 \prime}=0 \\
2 r \partial_{v} \mathbf{k}_{u}^{2 \prime \prime}-\partial_{u} \mathbf{k}_{v}^{2 \prime}+2 r \partial_{u} \mathbf{k}_{v}^{2 \prime \prime}+\partial_{v} \mathbf{k}_{u}^{2 \prime}+\partial_{v} \mathbf{k}_{u}^{2 \prime}-\partial_{u} \mathbf{k}_{v}^{2 \prime}=0 \\
-4 r^{2} \partial_{u} \mathbf{k}_{v}^{3 \prime \prime}+\partial_{v} \mathbf{k}_{v}^{2 \prime}-2 r \partial_{v} \mathbf{k}_{v}^{2 \prime \prime}+2 r \partial_{u} \mathbf{k}_{v}^{3 \prime}-10 r \partial_{u} \mathbf{k}_{v}^{3 \prime}-\partial_{v} \mathbf{k}_{v}^{2 \prime}=0 \\
2 r^{2} \partial_{u}^{2} \mathbf{k}_{v}^{3 \prime}-r \partial_{v}^{2} \mathbf{k}_{u}^{2 \prime}+2 r \partial_{u}^{2} \mathbf{k}_{v}^{3}-2 r \mathbf{k}_{v}^{2 \prime \prime}=0 \\
-4 r^{2} \mathbf{k}_{v}^{3 \prime \prime \prime}+\partial_{v}^{2} \mathbf{k}_{v}^{2 \prime}-2 r \partial_{u} \partial_{v} \mathbf{k}_{v}^{3 \prime}-16 r \mathbf{k}_{v}^{3 \prime \prime}-2 \partial_{u} \partial_{v} \mathbf{k}_{v}^{3}-8 \mathbf{k}_{v}^{3 \prime}=0 \\
-2 r \mathbf{k}_{u}^{2 \prime \prime \prime}-\partial_{u} \partial_{v} \mathbf{k}_{u}^{2 \prime}+\partial_{u}^{2} \mathbf{k}_{v}^{2 \prime}-4 \mathbf{k}_{u}^{2 \prime \prime}=0 .
\end{aligned}
$$

One observes that with the identification $\mathbf{k}_{u}^{2}=h_{u u}, \mathbf{k}_{v}^{2}=h_{u v}, \mathbf{k}_{v}^{3}=\frac{1}{2 r} h_{v v}$ the above equations are the same as that given in the equation (6.19) of [29] at critical value $\mu=1$. 
Similar observation has also been made in ref. [19] where the linearized equations of motion of MMG have been studied in the transverse traceless gauge. Note that eq. (5.4) contains seven equations. However, by the above identification the second and fifth equations in eq. (5.4) become identical. Note that this identification can also be understood as follows. In fact at the linearized level from the definition $g_{\mu \nu}=\eta_{a b} e_{\mu}^{a} e_{\nu}^{b}$ and in FG gauge one finds

$$
\frac{1}{r} h_{i j}=\mathbf{k}_{i}^{a} \bar{e}_{j a}+\bar{e}_{i}^{a} \mathbf{k}_{j a},
$$

which reduces to the above identification using eqs. (2.13) and (2.14).

By making use of this observation one can utilize the full solution of the TMG model obtained in [29] whose near boundary expansions are

$$
\begin{aligned}
\mathbf{k}_{u}^{2}= & \chi_{u}^{(0)} \log (r)+\varphi_{u}^{(0)}-\frac{1}{2} r \log (r) \partial_{u} \partial_{v} \chi_{u}^{(0)}+r\left(\left(\partial_{v}\right)^{-1} \partial_{u} \tilde{\varphi}_{v}^{(1)}+\frac{4 \gamma-3}{2} \partial_{u} \partial_{v} \chi_{u}^{(0)}\right)+\cdots, \\
\mathbf{k}_{v}^{3}= & \frac{1}{2 r}\left(\varphi_{v}^{(0)}+\frac{1}{2} r \log (r)\left(\partial_{u}\right)^{-1} \partial_{v}^{3} \chi_{u}^{(0)}\right. \\
& \left.\quad+r\left[\left(\partial_{u}\right)^{-1} \partial_{v} \tilde{\varphi}_{v}^{(1)}+\left(2 \gamma-1+2 \log \left(\frac{q}{2}\right)\right)\left(\partial_{u}\right)^{-1} \partial_{v}^{3} \chi_{u}^{(0)}\right]+\cdots\right), \\
\mathbf{k}_{v}^{2}= & \tilde{\varphi}_{v}^{(0)}-\frac{1}{2} r \log (r) \partial_{v}^{2} \chi_{u}^{(0)}+r \tilde{\varphi}_{v}^{(1)}+\cdots,
\end{aligned}
$$

where

$$
\tilde{\varphi}_{v}^{(1)}=-\frac{1}{2} \partial_{v}^{2} \chi_{u}^{(0)}-\frac{1}{2} \partial_{v}^{2} \varphi_{u}^{(0)}-\frac{1}{2} \partial_{u}^{2} \varphi_{v}^{(0)}+\partial_{u} \partial_{v} \tilde{\varphi}_{v}^{(0)} .
$$

Here $\chi_{u}^{(0)}, \varphi_{u}^{(0)}, \varphi_{v}^{(0)}, \tilde{\varphi}_{v}^{(0)}$ are arbitrary functions of the boundary coordinates $(u, v), q \equiv$ $\sqrt{-4 \partial_{u} \partial_{v}}$ and $\left(\partial_{i}\right)^{-1}$ denotes an integration with respect to $x^{i}$. Note that as far as the two-point function of stress tensor are concerned, the above explicit expansions of the full solution are enough.

We have now all ingredients to compute two-point functions of the stress tensor $T$ and its logarithmic partner $t$. To proceed one needs to express the one-point functions (5.2) as functions of sources. This can be achieved by substituting the full solution (5.6) into the equation (5.2) leading to

$$
\begin{aligned}
\left\langle T_{u u}\right\rangle & =\frac{1}{4 \mu G}\left(\partial_{v}\right)^{-1} \partial_{u}^{3} \varphi_{v}^{(0)}+\text { local, } \\
\left\langle T_{v v}\right\rangle & =-\frac{1}{4 G} \frac{\mu}{(\mu \sigma+2)^{2}}\left(\partial_{u}\right)^{-1} \partial_{v}^{3} \chi_{u}^{(0)}+\text { local, } \\
\left\langle T_{u v}\right\rangle & =\text { local, } \\
\left\langle t_{v v}\right\rangle & =\frac{1}{2 G} \frac{\mu}{(\mu \sigma+2)^{2}}\left(\left[-\frac{(5 \mu+\sigma+8)(\mu \sigma+2)^{2}}{16 \mu^{2}}-\frac{3}{2}+2 \gamma+2 \log \left(\frac{q}{2}\right)\right]\left(\partial_{u}\right)^{-1} \partial_{v}^{3} \chi_{u}^{(0)}\right. \\
& \left.-\frac{1}{2}\left(\partial_{u}\right)^{-1} \partial_{v}^{3} \varphi_{u}^{(0)}\right)+ \text { local. }
\end{aligned}
$$


From the identities

$$
\begin{aligned}
\frac{1}{\partial_{u} \partial_{v}} \delta^{2}(u, v) & =\frac{i}{2 \pi} \log \left(m^{2} u v\right), \\
\partial_{u}^{4}\left[\frac{i}{2 \pi} \log \left(m^{2} u v\right)\right] & =-\frac{3 i}{\pi} \frac{1}{u^{4}}, \\
\log (q)\left(\partial_{u}\right)^{-1} \partial_{v}^{3} \delta^{2}(u, v) & =\frac{i}{4 \pi}\left[-\frac{11}{v^{4}}+\frac{6}{v^{4}} \log \left(m^{2} u v\right)\right],
\end{aligned}
$$

one can compute derivatives of one-point functions with respect to the sources to find two-point functions as follows

$$
\begin{aligned}
\left\langle T_{u u} T_{u u}\right\rangle & =-\frac{3}{2 \mu G} \frac{1}{u^{4}}, \quad\left\langle T_{v v} t_{v v}\right\rangle=-\frac{3}{2 G} \frac{\mu}{(\mu \sigma+2)^{2}} \frac{1}{v^{4}}, \\
\left\langle t_{v v} t_{v v}\right\rangle & =\frac{3}{G} \frac{\mu}{(\mu \sigma+2)^{2}} \frac{\log \left(m^{2} u v\right)}{v^{4}}-\frac{1}{G} \frac{B}{v^{4}}
\end{aligned}
$$

and the correlation of other components are zero. Here $B$ is a constant which can be removed by a field redefinition, $t_{v v} \rightarrow t_{v v}-B \frac{(\mu \sigma+2)^{2}}{3 \mu} T_{v v}$.

It is shown that the asymptotic symmetry algebra of MMG model consists of two copies of the Virasoro algebra with central charges [8]

$$
c_{ \pm}=\frac{3}{2 G}\left(\sigma \pm \frac{1}{\mu}+\alpha C\right)
$$

which at critical point $\alpha=-\frac{2}{\mu}(1+\mu \sigma)$ they become

$$
c_{+}=0, \quad c_{-}=-\frac{3}{\mu G}
$$

which is consistent with the results given in eq. (5.10). Let us recall that in our notation the correlation functions of a LCFT are [33]

$$
\begin{aligned}
\left\langle T_{u u} T_{u u}\right\rangle & =\frac{c_{-}}{2 u^{4}}, \quad\left\langle T_{v v} T_{v v}\right\rangle=\frac{c_{+}}{2 v^{4}}, \quad\left\langle T_{v v} t_{v v}\right\rangle=\frac{b_{+}}{2 v^{4}}, \\
\left\langle t_{v v} t_{v v}\right\rangle & =-\frac{\log \left(m^{2} u v\right)\left[c_{+} \log \left(m^{2} u v\right)+2 b_{+}\right]}{2 v^{4}} .
\end{aligned}
$$

Comparing these expressions with that of eq. (5.10) one finds $b_{+}=-\frac{3}{G} \frac{\mu}{(\mu \sigma+2)^{2}}$ in agreement with [19].

\section{Conclusions}

In this paper we have studied holographic renormalization of recently proposed 3D gravitational theory known as MMG [8] in the dreibein formalism. In particular we have considered the model at the critical point $M=-1$ where it exhibits a new logarithmic solution.

To study holographic renormalization of the model for the logarithmic solution one needs to fix both the dreibein and its first radial derivative at the boundary. This, in turns, indicates that the dreibein and its derivative correspond to sources of two operators with 
dimension two in the holographic dual field theory. Indeed the dual field theory would be a logarithmic CFT and the corresponding dual operators are holographic stress tensor and its logarithmic partner. Using the holographic renormalization method we have evaluated the correlation functions among different components of these operators which confirm that the dual theory is indeed a logarithmic CFT. Actually these results together with the fact that one has vanishing central charge are common features of all three dimensional gravities that have critical point at which the models provide gravitational descriptions for logarithmic CFT's which are non-unitary.

It is important to note that in order to compute the correlation functions of holographic stress tensor and its logarithmic partner one has to make sure that the model has a well-defined variational principle and moreover the on-shell action for the corresponding solution is finite. In general to meet these two conditions one has to add proper boundary terms which include both the Gibbons-Hawking terms as well as certain counterterms. We have seen that as far as the model is concerned at critical point, the standard GibbonsHawking term is enough to have a well-defined variational principle. The full expressions for counterterms have also been presented.

It is also worth noting that using the holographic renormalization we have seen that the model at different limits $(\alpha=0, \mu \rightarrow \infty)$ and $(\alpha \neq 0, \mu \rightarrow \infty)$ has the same structure showing that adding the $\alpha$ term to the Einstein-Hilbert would not change the content of the theory.

\section{Acknowledgments}

We would like to thank Wout Merbis for useful discussions.

\section{A Explicit form of the action}

$$
\begin{aligned}
S_{M M G}= & \int_{\mathcal{M}} d^{3} x \epsilon^{\lambda \mu \nu}\left\{-\sigma e_{\lambda}^{a} d_{[\mu} \Omega_{\nu] a}-\frac{\sigma}{2} e_{\lambda}^{a} \varepsilon_{a b c} \Omega_{[\mu}^{b} \Omega_{\nu]}^{c}+\frac{\Lambda_{0}}{6} e_{\lambda}^{a} \varepsilon_{a b c} e_{[\mu}^{b} e_{\nu]}^{c}+h_{\lambda}^{a} d_{[\mu} e_{\nu] a}\right. \\
& +h_{\lambda}^{a} \varepsilon_{a b c} \Omega_{[\mu}^{b} e_{\nu]}^{c}+\frac{1}{2 \mu} \Omega_{\lambda}^{a} d_{[\mu} \Omega_{\nu] a}+\frac{1}{6 \mu} \Omega_{\lambda}^{a} \varepsilon_{a b c} \Omega_{[\mu}^{b} \Omega_{\nu]}^{c}+\sigma \alpha e_{\lambda}^{a} d_{[\mu} h_{\nu] a}+\sigma \alpha e_{\lambda}^{a} \varepsilon_{a b c} \Omega_{[\mu}^{b} h_{\nu]}^{c} \\
& -\frac{\alpha}{2}(1+\sigma \alpha) e_{\lambda}^{a} \varepsilon_{a b c} h_{[\mu}^{b} h_{\nu]}^{c}-\frac{\alpha}{2 \mu} \Omega_{\lambda}^{a} d_{[\mu} h_{\nu] a}-\frac{\alpha}{2 \mu} \Omega_{\lambda}^{a} \varepsilon_{a b c} \Omega_{[\mu}^{b} h_{\nu]}^{c}-\frac{\alpha}{2 \mu} h_{\lambda}^{a} d_{[\mu} \Omega_{\nu] a} \\
& \left.-\frac{\alpha}{4 \mu} h_{\lambda}^{a} \varepsilon_{a b c} \Omega_{[\mu}^{b} \Omega_{\nu]}^{c}+\frac{\alpha^{2}}{2 \mu} h_{\lambda}^{a} d_{[\mu} h_{\nu] a}+\frac{\alpha^{2}}{2 \mu} h_{\lambda}^{a} \varepsilon_{a b c} \Omega_{[\mu}^{b} h_{\nu]}^{c}-\frac{\alpha^{3}}{6 \mu} h_{\lambda}^{a} \varepsilon_{a b c} h_{[\mu}^{b} h_{\nu]}^{c}\right\}
\end{aligned}
$$

where in our notation, " $A_{[\mu} B_{\nu]}=A_{\mu} B_{\nu}-A_{\nu} B_{\mu} "$ and $" \epsilon \mu \nu "$ is " $\pm 1 " .{ }^{10}$ In our notation, from the first three terms in the above action we have

$$
S=\int_{\mathcal{M}} d^{3} x \epsilon^{\lambda \mu \nu}\left\{-\sigma e_{\lambda}^{a}\left(\partial_{\mu} \Omega_{\nu a}-\partial_{\nu} \Omega_{\mu a}\right)-\sigma \varepsilon_{a b c} e_{\lambda}^{a} \Omega_{\mu}^{b} \Omega_{\nu}^{c}+\frac{\Lambda_{0}}{3} \varepsilon_{a b c} e_{\lambda}^{a} e_{\mu}^{b} e_{\nu}^{c}\right\}
$$

which is the well-known action of three dimensional gravity [34].

\footnotetext{
${ }^{10}$ In our notation $\epsilon^{\lambda \mu \nu}=-e \tilde{\epsilon}^{\lambda \mu \nu}$ with $\tilde{\epsilon}^{\lambda \mu \nu}=\frac{1}{\sqrt{-g}}$.
} 


\section{B Divergent terms of on-shell action}

In this appendix we present explicit forms of divergent terms appearing in the on-shell action. Indeed, by making use of (2.25) the divergent terms of the on-shell action (4.3) are found ${ }^{11}$

$$
\begin{aligned}
\mathcal{S}_{1 / r}^{(0)}= & \frac{1}{2} \mu^{2} C^{3} \alpha^{3}+\frac{3}{2} \sigma \mu^{2} C^{2} \alpha^{2}+\frac{1}{4}\left(-3 C+6 C^{2} \mu^{2}\right) \alpha+\frac{1}{2} \sigma-\frac{1}{2} \Lambda_{0}, \\
\mathcal{S}_{\log ^{2}(r)}^{(1)}= & L L S \partial_{v}^{2} \chi_{u}^{(0)}, \\
\mathcal{S}_{\log (r)}^{(1)}= & \frac{1}{4 \mu^{2}(1+\sigma \alpha)^{2}}\left\{L L^{(1)} \partial_{v}^{2} \chi_{u}^{(0)}+2 L L^{(2)}\left(\partial_{u}^{2} \varphi_{v}^{(0)}+\partial_{v}^{2} \varphi_{u}^{(0)}-2 \partial_{u} \partial_{v} \tilde{\varphi}_{v}^{(0)}\right)\right\}, \\
\mathcal{S}_{1 / r}^{(1)}= & L R \tilde{\varphi}_{v}^{(0)} \\
\mathcal{S}_{\log ^{3}(r)}^{(2)}= & Q L T \chi_{u}^{(0)} \chi_{v}^{(1)}, \\
\mathcal{S}_{\log ^{2}(r)}^{(2)}= & \frac{1}{8 \mu(1+\sigma \alpha)^{2}}\left\{Q L S^{(1)}\left(\chi_{u}^{(0)} \varphi_{v}^{(2)}+\varphi_{u}^{(0)} \chi_{v}^{(1)}\right)+Q L S^{(2)} \chi_{u}^{(0)} \partial_{v}^{2} \tilde{\varphi}_{v}^{(0)}\right. \\
& \left.-\frac{1}{2} Q L S^{(3)} \chi_{u}^{(0)} \partial_{u} \partial_{v} \varphi_{v}^{(0)}\right\}, \\
\mathcal{S}_{\log (r) / r}^{(2)}= & Q L R \chi_{u}^{(0)} \varphi_{v}^{(0)}, \\
\mathcal{S}_{\log (r)}^{(2)}= & \frac{1}{(1+\sigma \alpha)^{4}}\left\{-\frac{1}{\mu^{3}} Q L^{(1)} \chi_{u}^{(0)} \chi_{v}^{(1)}+\frac{1}{2 \mu^{2}} Q L^{(2)}\left(-\chi_{u}^{(0)} \varphi_{v}^{(1)}+\varphi_{u}^{(0)} \chi_{v}^{(1)}\right)\right. \\
& +\frac{1}{4 \mu} Q L^{(3)} \varphi_{u}^{(0)} \varphi_{v}^{(1)}+\frac{1}{4 \mu} Q L^{(4)} \varphi_{v}^{(0)} \varphi_{u}^{(1)}+\frac{1}{4 \mu^{2}} Q L^{(5)} \chi_{u}^{(0)} \partial_{v}^{2} \tilde{\varphi}_{v}^{(0)} \\
& \left.+\frac{1}{4 \mu^{2}} Q L^{(6)} \chi_{u}^{(0)} \partial_{u} \partial_{v} \varphi_{v}^{(0)}+\frac{1}{4} Q L^{(7)}\left(\tilde{\varphi}_{v}^{(0)} \partial_{v}^{2} \varphi_{u}^{(0)}+\tilde{\varphi}_{v}^{(0)} \partial_{u}^{2} \varphi_{v}^{(0)}-\frac{1}{2} \tilde{\varphi}_{v}^{(0)} \partial_{u} \partial_{v} \tilde{\varphi}_{v}^{(0)}\right)\right\}, \\
\mathcal{S}_{1 / r}^{(2)}= & \frac{1}{(1+\sigma \alpha)^{2}}\left\{-\frac{1}{4 \mu^{2}} Q R^{(1)} \chi_{u}^{(0)} \varphi_{v}^{(0)}-\frac{1}{4} Q R^{(2)}\left(\varphi_{u}^{(0)} \varphi_{v}^{(0)}-\tilde{\varphi}_{v}^{(0) 2}\right)\right\}
\end{aligned}
$$

where

$$
\begin{aligned}
L L S= & \frac{1}{4} \mu^{2} C^{3} \alpha^{3}+\frac{3}{4} \sigma \mu^{2} C^{2} \alpha^{2}+\left(-\frac{3}{8} C+\frac{3}{4} \mu^{2} C^{2}\right) \alpha+\frac{1}{4 \mu}-\frac{3}{4} \sigma-\frac{1}{4} \Lambda_{0}, \\
L L^{(1)}= & 2 C^{3} \mu^{4} \alpha^{5}+\left(6 C^{2} \mu^{4} \sigma+4 C^{3} \mu^{4} \sigma\right) \alpha^{4}+\left(18 C^{2} \mu^{4}+2 C^{3} \mu^{4}-3 C \mu^{2}\right) \alpha^{3} \\
& +\left(-2 \Lambda_{0} \mu^{2}-6 \sigma C \mu^{2}+18 \sigma C^{2} \mu^{4}-6 \sigma \mu^{2}\right) \alpha^{2}+\left(6 C^{2} \mu^{4}-12 \mu^{2}-3 C \mu^{2}\right. \\
& \left.-4 \sigma \Lambda_{0} \mu^{2}-4\right) \alpha-6 \sigma \mu^{2}-2 \Lambda_{0} \mu^{2}, \\
L L^{(2)}= & C^{3} \mu^{4} \alpha^{5}+2 \sigma\left(C^{3}+\frac{3}{2} C^{2}\right) \mu^{4} \alpha^{4}+\mu^{2}\left(-\frac{3}{2} C+C^{3} \mu^{2}+9 C^{2} \mu^{2}\right) \alpha^{3} \\
& +\mu\left(1+9 \sigma C^{2} \mu^{3}-3 \sigma \mu-\Lambda_{0} \mu-3 \sigma C \mu\right) \alpha^{2} \\
& +\mu\left(3 C^{2} \mu^{3}-6 \mu-\frac{3}{2} C \mu-2 \sigma \Lambda_{0} \mu+2 \sigma\right) \alpha+\mu-3 \sigma \mu^{2}-\Lambda_{0} \mu^{2}, \\
L R= & C^{3} \mu^{2} \alpha^{3}+3 \sigma C^{2} \mu^{2} \alpha^{2}+\left(-\frac{3}{2} C+3 C^{2} \mu^{2}\right) \alpha+\sigma-\Lambda_{0},
\end{aligned}
$$

\footnotetext{
${ }^{11}$ To simplify these expressions we have assumed that $\sigma^{2}=1$. Moreover, throughout this paper we have also assumed that the $\chi_{u}^{(0)}$ is sufficiently small. Maple codes containing the calculations may be provided by the authors upon request.
} 
and

$$
\begin{aligned}
Q L T= & \frac{1}{6} C^{3} \mu^{2} \alpha^{3}+\left(\frac{1}{2} \sigma C^{2} \mu^{2}+\frac{1}{6} C^{2} \mu\right) \alpha^{2}+\left(\frac{1}{2} C^{2} \mu^{2}+\frac{1}{3} \sigma C \mu-\frac{1}{4} C\right) \alpha \\
& -\frac{1}{6} \sigma-\frac{1}{6} \Lambda_{0}-\frac{1}{6 \mu}+\frac{1}{3} C \mu, \\
Q L S^{(1)}= & 2 C^{3} \mu^{3} \alpha^{5}+\left(4 \sigma C^{3} \mu^{3}+2 C^{2} \mu^{2}+6 \sigma C^{2} \mu^{3}\right) \alpha^{4}+\left(-3 C+18 C^{2} \mu^{3}\right. \\
& \left.+4 \sigma C \mu^{2} \mu+4 \sigma \mu^{2} C^{2}+2 \mu^{3} C^{3}\right) \alpha^{3}+\left(-2+12 C \mu^{2}-2 \sigma \mu+18 \sigma \mu^{3} C^{2}\right. \\
& \left.+2 C^{2} \mu^{2}-6 \sigma \mu C-2 \mu \Lambda_{0}\right) \alpha^{2}+\left(12 \sigma C \mu^{2}-4 \sigma-4 \mu-3 C \mu-4 \sigma \mu \Lambda_{0}\right. \\
& \left.+6 \mu^{3} C^{2}\right) \alpha-2-2 \sigma \mu-2 \mu \Lambda_{0}+4 C \mu^{2}, \\
Q L S^{(2)}= & 2 C^{3} \mu^{3} \alpha^{5}+\left(4 \sigma C^{3} \mu^{3}+6 \sigma C^{2} \mu^{3}\right) \alpha^{4}+\left(2 \mu^{3} C^{3}-C \mu+18 C^{2} \mu^{3}\right) \alpha^{3}
\end{aligned}
$$$$
+\left(18 \sigma C^{2} \mu^{3}-2 \sigma \mu-2 \Lambda_{0} \mu-2 \sigma C \mu\right) \alpha^{2}+\left(-4 \mu-C \mu-4 \sigma \mu \Lambda_{0}+6 C^{2} \mu^{3}\right) \alpha
$$$$
-2 \mu \Lambda_{0}-2 \sigma \mu \text {, }
$$

$$
\begin{aligned}
Q L S^{(3)}= & 2 C^{3} \mu^{3} \alpha^{5}+\left(4 \sigma \mu^{3} C^{3}-2 C^{2} \mu^{2}+6 \sigma \mu^{3} C^{2}\right) \alpha^{4}+\left(C \mu+18 \mu^{3} C^{2}-4 \sigma C \mu^{2}\right. \\
& \left.-4 \sigma \mu^{2} C^{2}+2 \mu^{3} C^{3}\right) \alpha^{3}+\left(2-12 C \mu^{2}-2 \sigma \mu+18 \sigma C^{2} \mu^{3}-2 C^{2} \mu^{2}+2 \sigma \mu C\right. \\
& \left.-2 \mu \Lambda_{0}\right) \alpha^{2}+\left(-12 \sigma C \mu^{2}+4 \sigma-4 \mu+C \mu-4 \sigma \mu \Lambda_{0}+6 \mu^{3} C^{2}\right) \alpha+2-2 \sigma \mu \\
& -2 \mu \Lambda_{0}-4 C \mu^{2}, \\
Q L R= & -\frac{1}{2} \mu^{2} C^{3} \alpha^{3}-\frac{3}{2} \sigma \mu^{2} C^{2} \alpha^{2}-\frac{1}{2}\left(-\frac{3}{2} C+3 C^{2} \mu^{2}\right) \alpha-\frac{1}{2} \sigma+\frac{1}{2} \Lambda_{0},
\end{aligned}
$$$$
Q L^{(1)}=C \mu^{3} \alpha^{5}+\left(-2 \sigma \mu^{3}-4 \mu^{2}+4 C \mu^{2}+4 \sigma C \mu^{3}\right) \alpha^{4}+\left(8 \sigma C \mu^{2}-8 \sigma \mu^{2}-16 \mu\right.
$$$$
\left.+8 C \mu+6 C \mu^{3}-8 \mu^{3}\right) \alpha^{3}+\left(4 \sigma C \mu^{3}+4 C \mu^{2}-8 \mu^{2}-12 \sigma \mu^{3}-8-24 \sigma \mu\right) \alpha^{2}
$$$$
+\left(-8 \sigma \mu^{2}-8 \mu^{3}-8 \mu+C \mu^{3}\right) \alpha-2 \sigma \mu^{3}-4 \mu^{2},
$$$$
Q L^{(2)}=C^{2} \mu^{3} \alpha^{6}+\left(4 \sigma \mu^{3} C^{2}-2 C \mu^{2}+4 C^{2} \mu^{2}+2 \sigma C \mu^{3}\right) \alpha^{5}+\left(10 C \mu^{3}-2 \sigma \mu^{2}\right.
$$$$
\left.+6 C^{2} \mu^{3}-3 \mu+8 \sigma \mu^{2} C^{2}\right) \alpha^{4}+\left(12 C \mu^{2}+20 \sigma C \mu^{3}-12 \sigma \mu-8 \mu^{2}-6+4 C^{2} \mu^{2}\right.
$$$$
\left.+4 \sigma C^{2} \mu^{3}\right) \alpha^{3}+\left(C^{2} \mu^{3}+16 \sigma C \mu^{2}-12 \sigma \mu^{2}+20 C \mu^{3}-18 \mu-12 \sigma\right) \alpha^{2}
$$$$
+\left(-8 \mu^{2}-6+10 \sigma C \mu^{3}+6 C \mu^{2}-12 \sigma \mu\right) \alpha+2 C \mu^{3}-3 \mu-2 \sigma \mu^{2} \text {, }
$$

$Q L^{(3)}=2 C^{3} \mu^{3} \alpha^{7}+\left(2 C^{2} \mu^{2}+8 \sigma C^{3} \mu^{3}+6 \sigma C^{2} \mu^{3}\right) \alpha^{6}+\left(30 C^{2} \mu^{3}+12 C^{3} \mu^{3}-3 C \mu\right.$

$\left.+8 \sigma C^{2} \mu^{2}+4 \sigma C \mu^{2}\right) \alpha^{5}+\left(8 \sigma C^{3} \mu^{3}+20 C \mu^{2}+12 C^{2} \mu^{2}-12 \sigma C \mu+60 \sigma C^{2} \mu^{3}\right.$

$\left.-2 \sigma \mu-2 \mu \Lambda_{0}-2\right) \alpha^{4}+\left(-8 \mu-8 \sigma+2 C^{3} \mu^{3}-18 C \mu+40 \sigma C \mu^{2}+60 C^{2} \mu^{3}\right.$

$\left.-8 \sigma \mu \Lambda_{0}+8 \sigma C^{2} \mu^{2}\right) \alpha^{3}+\left(-12-12 \mu \Lambda_{0}+2 C^{2} \mu^{2}-12 \sigma \mu C+40 C \mu^{2}+30 \sigma C^{2} \mu^{3}\right.$

$-12 \sigma \mu) \alpha^{2}+\left(-8 \mu-8 \sigma+20 \sigma C \mu^{2}-3 C \mu+6 C^{2} \mu^{3}-8 \sigma \mu \Lambda_{0}\right) \alpha-2-2 \sigma \mu$

$-2 \mu \Lambda_{0}+4 C \mu^{2}$,

$Q L^{(4)}=2 C^{3} \mu^{3} \alpha^{7}+\left(-2 C^{2} \mu^{2}+8 \sigma C^{3} \mu^{3}+6 \sigma C^{2} \mu^{3}\right) \alpha^{6}+\left(30 C^{2} \mu^{3}+12 C^{3} \mu^{3}+C \mu\right.$

$\left.-8 \sigma C^{2} \mu^{2}-4 \sigma C \mu^{2}\right) \alpha^{5}+\left(8 \sigma C^{3} \mu^{3}-20 C \mu^{2}-12 C^{2} \mu^{2}+4 \sigma C \mu+60 \sigma C^{2} \mu^{3}\right.$

$\left.-2 \sigma \mu-2 \mu \Lambda_{0}+2\right) \alpha^{4}+\left(-8 \mu+8 \sigma+2 C^{3} \mu^{3}+6 C \mu-40 \sigma C \mu^{2}+60 C^{2} \mu^{3}\right.$

$\left.-8 \sigma \mu \Lambda_{0}-8 \sigma C^{2} \mu^{2}\right) \alpha^{3}+\left(12-12 \mu \Lambda_{0}-2 C^{2} \mu^{2}+4 \sigma \mu C-40 C \mu^{2}+30 \sigma C^{2} \mu^{3}\right.$

$-12 \sigma \mu) \alpha^{2}+\left(-8 \mu+8 \sigma-20 \sigma C \mu^{2}+C \mu+6 C^{2} \mu^{3}-8 \sigma \mu \Lambda_{0}\right) \alpha+2-2 \sigma \mu$

$-2 \mu \Lambda_{0}-4 C \mu^{2}$, 


$$
\begin{aligned}
Q L^{(5)}= & 2 C^{3} \mu^{4} \alpha^{7}+\left(6 \sigma C^{2} \mu^{4}+8 \sigma C^{3} \mu^{4}\right) \alpha^{6}+\left(12 C^{3} \mu^{4}+8 C^{2} \mu^{2}+30 C^{2} \mu^{4}-3 C \mu^{2}\right) \alpha^{5} \\
& +\left(16 \sigma C^{2} \mu^{2}+4 \sigma C \mu^{2}+8 \sigma C^{3} \mu^{4}-2 \mu^{2} \Lambda_{0}-4 \mu-6 \sigma \mu^{2}+60 \sigma C^{2} \mu^{4}\right) \alpha^{4} \\
& +\left(-20+30 C \mu^{2}-16 \sigma \mu+2 C^{3} \mu^{4}-24 \mu^{2}+60 C^{2} \mu^{4}-8 \sigma \mu^{2} \Lambda_{0}+8 C^{2} \mu^{2}\right) \alpha^{3} \\
& +\left(-24 \mu-40 \sigma+30 \sigma C^{2} \mu^{4}-12 \mu^{2} \Lambda_{0}+36 \sigma C \mu^{2}-36 \sigma \mu^{2}\right) \alpha^{2}+(-16 \sigma \mu-20 \\
& \left.-24 \mu^{2}+6 C^{2} \mu^{4}+13 C \mu^{2}-8 \sigma \mu^{2} \Lambda_{0}\right) \alpha-4 \mu-2 \mu^{2} \Lambda_{0}-6 \sigma \mu^{2}, \\
Q L^{(6)}= & C^{2} \mu^{3} \alpha^{6}+\left(-4 C^{2} \mu^{2}+4 \sigma C^{2} \mu^{3}+2 \sigma C \mu^{3}\right) \alpha^{5}+\left(2 \sigma \mu^{2}-8 \sigma C^{2} \mu^{2}+\mu+6 C^{2} \mu^{3}\right. \\
& \left.-8 \sigma C \mu^{2}+10 C \mu^{3}\right) \alpha^{4}+\left(8 \mu^{2}+6+4 \sigma \mu-4 C^{2} \mu^{2}+4 \sigma C^{2} \mu^{3}+20 \sigma C \mu^{3}\right. \\
& \left.-24 C \mu^{2}\right) \alpha^{3}+\left(C^{2} \mu^{3}+20 C \mu^{3}+12 \sigma \mu^{2}+12 \sigma-24 \sigma C \mu^{2}+6 \mu\right) \alpha^{2}+\left(10 \sigma C \mu^{3}\right. \\
& \left.+6-8 C \mu^{2}+8 \mu^{2}+4 \sigma \mu\right) \alpha+2 \sigma \mu^{2}+\mu+2 C \mu^{3}, \\
Q L^{(7)}= & 2 C^{3} \mu^{2} \alpha^{7}+\left(8 \sigma C^{3} \mu^{2}+6 \sigma C^{2} \mu^{2}\right) \alpha^{6}+\left(-C+30 C^{2} \mu^{2}+12 C^{3} \mu^{2}\right) \alpha^{5}+\left(8 \sigma C^{3} \mu^{2}\right. \\
& \left.-2 \Lambda_{0}+60 \sigma C^{2} \mu^{2}-4 \sigma C-2 \sigma\right) \alpha^{4}+\left(-6 C+2 C^{3} \mu^{2}+60 C^{2} \mu^{2}-8 \sigma \Lambda_{0}-8\right) \alpha^{3} \\
& +\left(-4 \sigma C-12 \sigma-12 \Lambda_{0}+30 \sigma C^{2} \mu^{2}\right) \alpha^{2}+\left(-8-8 \sigma \Lambda_{0}-C+6 C^{2} \mu^{2}\right) \alpha-2 \Lambda_{0} \\
& -2 \sigma, \\
Q R^{(1)}= & 2 C^{3} \mu^{4} \alpha^{5}+\left(4 \sigma C^{3} \mu^{4}+6 \sigma C^{2} \mu^{4}-2 C^{2} \mu^{3}\right) \alpha^{4}+\left(2 C^{3} \mu^{4}-8 C^{2} \mu^{2}-4 \sigma C^{2} \mu^{3}\right. \\
& \left.-4 \sigma C \mu^{3}+18 C^{2} \mu^{4}+5 C \mu^{2}\right) \alpha^{3}+\left(8 C \mu-2 C^{2} \mu^{3}+18 \sigma C^{2} \mu^{4}-12 C \mu^{3}-2 \mu^{2} \Lambda_{0}\right. \\
& \left.-2 \mu-6 \sigma C \mu^{2}-2 \sigma \mu^{2}\right) \alpha^{2}+\left(-4+12 \sigma \mu-4 \mu^{2}-11 C \mu^{2}-4 \sigma \mu^{2} \Lambda_{0}-12 \sigma C \mu^{3}\right. \\
& \left.+6 C^{2} \mu^{4}\right) \alpha-2 \mu-2 \sigma \mu^{2}-4 C \mu^{3}-2 \mu^{2} \Lambda_{0}, \\
Q R^{(2)}= & 2 C^{3} \mu^{2} \alpha^{5}+\left(6 \sigma C^{2} \mu^{2}+4 \sigma C^{3} \mu^{2}\right) \alpha^{4}+\left(2 C^{3} \mu^{2}-3 C+18 C^{2} \mu^{2}\right) \alpha^{3}+\left(18 \sigma C^{2} \mu^{2}\right. \\
& \left.-6 \sigma C-2 \Lambda_{0}+2 \sigma\right) \alpha^{2}+\left(-3 C+4-4 \sigma \Lambda_{0}+6 C^{2} \mu^{2}\right) \alpha+2 \sigma-2 \Lambda_{0} .
\end{aligned}
$$

\section{Coefficients in counterterm action}

In this appendix, explicit values of the coefficients appearing in the action of counterterms for two cases $\sigma=-1$ and $\sigma=1$ are presented.

$$
\begin{array}{rlrl}
\mathbf{C . 1} \boldsymbol{\sigma} & =-\mathbf{1} \\
a_{1} & =-\frac{15 \mu^{3}-87 \mu^{2}+16 \mu+60}{32 \mu^{2}}, & a_{2} & =a_{4}=\frac{5 \mu-9}{16 \mu}, \\
a_{3} & =-\frac{1}{\mu}, & a_{5} & =\frac{\mu^{3}-9 \mu^{2}+48 \mu-60}{32 \mu^{2}}, \\
a_{6} & =\frac{(5 \mu-1)(\mu-2)^{2}}{16 \mu^{2}}, & a_{7} & =-\frac{3 \mu-1}{64 \mu}, \\
b_{1} & =-\frac{9 \mu^{3}-47 \mu^{2}+88 \mu-52}{16 \mu^{2}}, & b_{2} & =\frac{3 \mu^{3}-16 \mu^{2}+34 \mu-22}{8 \mu^{2}}, \\
b_{3} & =-\frac{1}{2} b_{4}=\frac{1}{4} b_{8}=4 c_{5}=\frac{\mu-1}{8 \mu}, & b_{5} & =-\frac{4 \mu^{3}-21 \mu^{2}+40 \mu-24}{8 \mu^{2}}, \\
b_{6} & =\frac{4 \mu^{3}-21 \mu^{2}+38 \mu-22}{8 \mu^{2}}, & b_{7} & =\frac{(\mu-2)^{2}\left(9 \mu^{3}-41 \mu^{2}+76 \mu-48\right)}{32 \mu^{3}}, \\
c_{1} & =-4 c_{2}=-\frac{4}{3} c_{3}=\frac{(\mu-1)(\mu-2)^{2}}{16 \mu^{2}}, & c_{4} & =6 d_{1}=-\frac{(\mu-1)(\mu-2)^{4}}{64 \mu^{3}} .
\end{array}
$$




$$
\begin{array}{rlrl}
\mathbf{C . 2} \boldsymbol{\sigma} & =1 \mu^{3}+45 \mu^{2}-32 \mu-68 \\
a_{1} & =\frac{5 \mu^{2}}{12}, & a_{2} & =a_{5}=-\frac{5 \mu+9}{16 \mu}, \\
a_{3} & =-\frac{(5 \mu+1)(\mu+2)^{2}}{16 \mu^{2}}, & a_{4} & =-\frac{1}{\mu}, \\
a_{6} & =-\frac{11 \mu^{3}+51 \mu^{2}+96 \mu+68}{32 \mu^{2}}, & a_{7} & =-\frac{3 \mu^{3}+15 \mu^{2}+44 \mu+40}{128 \mu(\mu+2)^{2}}, \\
b_{1} & =-\frac{9 \mu^{3}+29 \mu^{2}+52 \mu+40}{32 \mu^{2}}, & b_{2} & =-\frac{(\mu+1)\left(3 \mu^{2}+20 \mu+40\right)}{32 \mu^{2}}, \\
b_{3} & =-\frac{1}{2} b_{4}=\frac{1}{4} b_{7}=4 c_{5}=-\frac{\mu+1}{8 \mu}, & b_{5} & =\frac{(\mu+1)\left(7 \mu^{2}+36 \mu+48\right)}{32 \mu^{2}} \\
b_{6} & =\frac{11 \mu^{3}+39 \mu^{2}+76 \mu+56}{32 \mu^{2}}, & b_{8} & =-\frac{9 \mu^{3}+41 \mu^{2}+76 \mu+48}{16 \mu(\mu+2)^{2}}, \\
c_{1} & =-4 c_{2}=-\frac{4}{3} c_{3}=-\frac{(\mu+1)(\mu+2)^{2}}{16 \mu^{2}}, & c_{4} & =6 d_{1}=\frac{(\mu+1)(\mu+2)^{4}}{64 \mu^{3}} .
\end{array}
$$

\section{Local finite terms in on-shell action}

In this appendix, explicit forms of the local terms in the renormalized on-shell action for two cases $\sigma=-1$ and $\sigma=1$ are provided.

$$
\begin{aligned}
\mathbf{D . 1} \boldsymbol{\sigma}=-\mathbf{1} & \begin{aligned}
\left.S_{\text {ren }}\right|_{\text {local }}=\int d^{2} x\{ & \frac{2\left(\mu^{3}-4 \mu^{2}+4\right)}{\mu(\mu-2)^{2}} \chi_{u}^{(0)} \partial_{u} \partial_{v} \varphi_{v}^{(0)}-\frac{29 \mu^{3}-141 \mu^{2}+240 \mu-140}{4 \mu(\mu-2)^{2}} \chi_{u}^{(0)} \partial_{v}^{2} \tilde{\varphi}_{v}^{(0)} \\
& -\frac{15 \mu-19}{4 \mu} \tilde{\varphi}_{v}^{(0)} \partial_{v}^{2} \varphi_{u}^{(0)}-\frac{15 \mu-19}{4 \mu} \tilde{\varphi}_{v}^{(0)} \partial_{u}^{2} \varphi_{v}^{(0)} \\
& \left.+\frac{19 \mu-27}{4 \mu} \tilde{\varphi}_{v}^{(0)} \partial_{u} \partial_{v} \tilde{\varphi}_{v}^{(0)}-\frac{\mu+7}{4 \mu} \varphi_{v}^{(0)} \partial_{u} \partial_{v} \varphi_{u}^{(0)}\right\} .
\end{aligned}
\end{aligned}
$$

$$
\begin{aligned}
& \begin{array}{l}
\text { D.2 } \\
\left.\quad S_{\text {ren }}\right|_{\text {local }}=1 \\
=
\end{array} d^{2} x\left\{-\frac{2\left(\mu^{3}+4 \mu^{2}-4\right)}{\mu(\mu+2)^{2}} \chi_{u}^{(0)} \partial_{u} \partial_{v} \varphi_{v}^{(0)}+\frac{39 \mu^{3}+183 \mu^{2}+288 \mu+148}{4 \mu(\mu+2)^{2}} \chi_{u}^{(0)} \partial_{v}^{2} \tilde{\varphi}_{v}^{(0)}\right. \\
& +\frac{15 \mu+19}{4 \mu} \tilde{\varphi}_{v}^{(0)} \partial_{v}^{2} \varphi_{u}^{(0)}+\frac{15 \mu+19}{4 \mu} \tilde{\varphi}_{v}^{(0)} \partial_{u}^{2} \varphi_{v}^{(0)} \\
& -\frac{7 \mu^{3}+36 \mu^{2}+65 \mu+39}{\mu(\mu+2)^{2}} \tilde{\varphi}_{v}^{(0)} \partial_{u} \partial_{v} \tilde{\varphi}_{v}^{(0)} \\
& \left.-\frac{2 \mu^{3}+11 \mu^{2}+25 \mu+19}{\mu(\mu+2)^{2}} \varphi_{v}^{(0)} \partial_{u} \partial_{v} \varphi_{u}^{(0)}\right\} \text {. }
\end{aligned}
$$




\section{E Local terms in one-point functions}

In this appendix explicit forms of the local terms in one-point functions are given. The contributions of these local terms to the two-point functions are contact terms. It is important to note that these local terms contribute to the boundary charges such as mass, angular momentum and entropy of black hole solutions.

$$
\begin{aligned}
& \begin{array}{l}
\text { E.1 } \quad \boldsymbol{\sigma}=-\mathbf{1} \\
\left.\left\langle T_{\text {uu }}\right\rangle\right|_{\text {local }}=\frac{1}{8 G}\left(\frac{2\left(\mu^{3}-4 \mu^{2}+4\right)}{\mu(\mu-2)^{2}} \partial_{u} \partial_{v} \chi_{u}^{(0)}-\frac{15 \mu-19}{4 \mu} \partial_{u}^{2} \tilde{\varphi}_{v}^{(0)}-\frac{\mu+7}{4 \mu} \partial_{u} \partial_{v} \varphi_{u}^{(0)}\right)
\end{array} \\
& \left\langle T_{v v}\right\rangle_{\text {local }}=\frac{1}{8 G}\left(-\frac{15 \mu-19}{4 \mu} \partial_{v}^{2} \tilde{\varphi}_{v}^{(0)}-\frac{\mu+7}{4 \mu} \partial_{u} \partial_{v} \varphi_{v}^{(0)}\right) \\
& \left.\left\langle T_{u v}\right\rangle\right|_{\text {local }}=\frac{1}{8 G}\left(-\frac{15 \mu-19}{4 \mu} \partial_{v}^{2} \varphi_{u}^{(0)}-\frac{15 \mu-19}{4 \mu} \partial_{u}^{2} \varphi_{v}^{(0)}+\frac{19 \mu-27}{2 \mu} \partial_{u} \partial_{v} \tilde{\varphi}_{v}^{(0)}\right. \\
& \left.-\frac{29 \mu^{3}-141 \mu^{2}+240 \mu-140}{4 \mu(\mu-2)^{2}} \partial_{v}^{2} \chi_{u}^{(0)}\right) \text {, } \\
& \left.\left\langle t_{v v}\right\rangle\right|_{\text {local }}=-\frac{1}{8 G}\left(\frac{2\left(\mu^{3}-4 \mu^{2}+4\right)}{\mu(\mu-2)^{2}} \partial_{u} \partial_{v} \varphi_{v}^{(0)}-\frac{29 \mu^{3}-141 \mu^{2}+240 \mu-140}{4 \mu(\mu-2)^{2}} \partial_{v}^{2} \tilde{\varphi}_{v}^{(0)}\right) .
\end{aligned}
$$

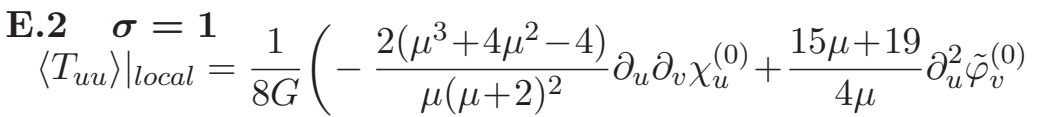

$$
\begin{aligned}
& \left.-\frac{2 \mu^{3}+11 \mu^{2}+25 \mu+19}{\mu(\mu+2)^{2}} \partial_{u} \partial_{v} \varphi_{u}^{(0)}\right), \\
& \left.\left\langle T_{v v}\right\rangle\right|_{\text {local }}=\frac{1}{8 G}\left(\frac{15 \mu+19}{4 \mu} \partial_{v}^{2} \tilde{\varphi}_{v}^{(0)}-\frac{2 \mu^{3}+11 \mu^{2}+25 \mu+19}{\mu(\mu+2)^{2}} \partial_{u} \partial_{v} \varphi_{v}^{(0)}\right), \\
& \left.\left\langle T_{u v}\right\rangle\right|_{\text {local }}=\frac{1}{8 G}\left(\frac{15 \mu+19}{4 \mu} \partial_{v}^{2} \varphi_{u}^{(0)}+\frac{15 \mu+19}{4 \mu} \partial_{u}^{2} \varphi_{v}^{(0)}-2 \frac{7 \mu^{3}+36 \mu^{2}+65 \mu+39}{\mu(\mu+2)^{2}} \partial_{u} \partial_{v} \tilde{\varphi}_{v}^{(0)}\right. \\
& \left.+\frac{39 \mu^{3}+183 \mu^{2}+288 \mu+148}{4 \mu(\mu+2)^{2}} \partial_{v}^{2} \chi_{u}^{(0)}\right) \\
& \left\langle\left. t_{v v}\right|_{\text {local }}=-\frac{1}{8 G}\left(-\frac{2\left(\mu^{3}+4 \mu^{2}-4\right)}{\mu(\mu+2)^{2}} \partial_{u} \partial_{v} \varphi_{v}^{(0)}+\frac{39 \mu^{3}+183 \mu^{2}+288 \mu+148}{4 \mu(\mu+2)^{2}} \partial_{v}^{2} \tilde{\varphi}_{v}^{(0)}\right) .\right.
\end{aligned}
$$

Open Access. This article is distributed under the terms of the Creative Commons Attribution License (CC-BY 4.0), which permits any use, distribution and reproduction in any medium, provided the original author(s) and source are credited.

\section{References}

[1] S. Deser, R. Jackiw and S. Templeton, Three-Dimensional Massive Gauge Theories, Phys. Rev. Lett. 48 (1982) 975 [INSPIRE].

[2] S. Deser, R. Jackiw and S. Templeton, Topologically Massive Gauge Theories, Annals Phys. 140 (1982) 372 [Erratum ibid. 185 (1988) 406] [Annals Phys. 281 (2000) 409] [INSPIRE].

[3] K.A. Moussa, G. Clement and C. Leygnac, The black holes of topologically massive gravity, Class. Quant. Grav. 20 (2003) L277 [gr-qc/0303042] [INSPIRE]. 
[4] A. Bouchareb and G. Clement, Black hole mass and angular momentum in topologically massive gravity, Class. Quant. Grav. 24 (2007) 5581 [arXiv:0706. 0263] [INSPIRE].

[5] D. Anninos, W. Li, M. Padi, W. Song and A. Strominger, Warped AdS $S_{3}$ Black Holes, JHEP 03 (2009) 130 [arXiv:0807.3040] [INSPIRE].

[6] K.A. Moussa, G. Clement, H. Guennoune and C. Leygnac, Three-dimensional Chern-Simons black holes, Phys. Rev. D 78 (2008) 064065 [arXiv: 0807.4241] [INSPIRE].

[7] W. Li, W. Song and A. Strominger, Chiral Gravity in Three Dimensions, JHEP 04 (2008) 082 [arXiv:0801.4566] [INSPIRE].

[8] E. Bergshoeff, O. Hohm, W. Merbis, A.J. Routh and P.K. Townsend, Minimal Massive 3D Gravity, Class. Quant. Grav. 31 (2014) 145008 [arXiv:1404.2867] [InSPIRE].

[9] M. Henningson and K. Skenderis, The Holographic Weyl anomaly, JHEP 07 (1998) 023 [hep-th/9806087] [INSPIRE].

[10] M. Bañados and F. Mendez, A note on covariant action integrals in three-dimensions, Phys. Rev. D 58 (1998) 104014 [hep-th/9806065] [INSPIRE].

[11] O. Mišković and R. Olea, On boundary conditions in three-dimensional AdS gravity, Phys. Lett. B 640 (2006) 101 [hep-th/0603092] [INSPIRE].

[12] M. Blagojevic, B. Cvetkovic, O. Mišković and R. Olea, Holography in 3D AdS gravity with torsion, JHEP 05 (2013) 103 [arXiv: 1301.1237] [INSPIRE].

[13] D. Grumiller and W. Merbis, Free energy of topologically massive gravity and flat space holography, arXiv: 1509.08505 [INSPIRE].

[14] M. Alishahiha and A. Naseh, Holographic renormalization of new massive gravity, Phys. Rev. D 82 (2010) 104043 [arXiv: 1005.1544] [INSPIRE].

[15] F. Loran, Holographic anomaly in 3d f(Ric) gravity, Class. Quant. Grav. 30 (2013) 195010 [arXiv: 1302.4584] [INSPIRE].

[16] H.R. Afshar, E.A. Bergshoeff and W. Merbis, Extended massive gravity in three dimensions, JHEP 08 (2014) 115 [arXiv: 1405.6213] [INSPIRE].

[17] A.S. Arvanitakis, A.J. Routh and P.K. Townsend, Matter coupling in 3D 'minimal massive gravity', Class. Quant. Grav. 31 (2014) 235012 [arXiv:1407.1264] [INSPIRE].

[18] B. Tekin, Minimal Massive Gravity: Conserved Charges, Excitations and the Chiral Gravity Limit, Phys. Rev. D 90 (2014) 081701 [arXiv: 1409.5358] [inSPIRE].

[19] M. Alishahiha, M.M. Qaemmaqami, A. Naseh and A. Shirzad, On 3D Minimal Massive Gravity, JHEP 12 (2014) 033 [arXiv:1409.6146] [INSPIRE].

[20] A.S. Arvanitakis and P.K. Townsend, Minimal Massive 3D Gravity Unitarity Redux, Class. Quant. Grav. 32 (2015) 085003 [arXiv:1411.1970] [inSPIRE].

[21] G. Giribet and Y. Vásquez, Minimal Log Gravity, Phys. Rev. D 91 (2015) 024026 [arXiv: 1411.6957] [INSPIRE].

[22] M.R. Setare, On the Generalized Minimal Massive Gravity, Nucl. Phys. B 898 (2015) 259 [arXiv: 1412.2151] [INSPIRE].

[23] A.S. Arvanitakis, On Solutions of Minimal Massive 3D Gravity, Class. Quant. Grav. 32 (2015) 115010 [arXiv:1501.01808] [INSPIRE]. 
[24] E. Altas and B. Tekin, Exact solutions and the consistency of 3D minimal massive gravity, Phys. Rev. D 92 (2015) 025033 [arXiv: 1503.04726] [INSPIRE].

[25] D. Mahdavian Yekta, Hamiltonian formalism of Minimal Massive Gravity, Phys. Rev. D 92 (2015) 064044 [arXiv: 1503.08343] [InSPIRE].

[26] N.S. Deger and O. Sarioglu, Kundt solutions of Minimal Massive 3D Gravity, Phys. Rev. D 92 (2015) 104015 [arXiv: 1505.03387] [INSPIRE].

[27] D. Grumiller and I. Sachs, $A d S_{3} / L C F T_{2}$ - Correlators in Cosmological Topologically Massive Gravity, JHEP 03 (2010) 012 [arXiv:0910.5241] [INSPIRE].

[28] W. Merbis, Chern-Simons-like Theories of Gravity, arXiv:1411.6888 [INSPIRE].

[29] K. Skenderis, M. Taylor and B.C. van Rees, Topologically Massive Gravity and the AdS/CFT Correspondence, JHEP 09 (2009) 045 [arXiv:0906.4926] [INSPIRE].

[30] S. de Haro, S.N. Solodukhin and K. Skenderis, Holographic reconstruction of space-time and renormalization in the AdS/CFT correspondence, Commun. Math. Phys. 217 (2001) 595 [hep-th/0002230] [INSPIRE].

[31] P. Kraus and F. Larsen, Holographic gravitational anomalies, JHEP 01 (2006) 022 [hep-th/0508218] [INSPIRE].

[32] S. Detournay, D. Grumiller, F. Schöller and J. Simón, Variational principle and one-point functions in three-dimensional flat space Einstein gravity, Phys. Rev. D 89 (2014) 084061 [arXiv: 1402.3687] [INSPIRE].

[33] V. Gurarie and A.W.W. Ludwig, Conformal field theory at central charge $c=0$ and two-dimensional critical systems with quenched disorder, In From fields to strings, vol. 2, M. Shifman et al. eds., pg. 1384-1440, hep-th/0409105 [INSPIRE].

[34] E. Witten, (2+1)-Dimensional Gravity as an Exactly Soluble System, Nucl. Phys. B 311 (1988) 46 [INSPIRE]. 